\title{
NUCLEAR MEDICINE IN THE PEDIATRIC FIELD
}

Barone Sebastiano ${ }^{1}$, Pagliuso Serena ${ }^{2}$, Pagliuso Luana ${ }^{3}$

${ }^{1}$ Docente di tecniche diagnostiche medico nucleari, Università degli studi di Roma La Sapienza

2 Infermiera, Ospedale Santa Scolastica di Cassino

${ }^{3}$ TSRM, SS.Trinità di Cagliari

KEYWORDS: pediatric nuclear medicine, pediatric scintigraphy, pediatric radiology,

\section{ABSTRACT}

The work presented describes the main ways of preparing radiopharmaceuticals and carrying out nuclear-medical examinations in pediatric patients. Through the differences in the execution of the tests, the importance of dosimetry is revealed, i.e. the diagnostic reference levels, in which the percentage of activity that must be administered to the child on the basis of that introduced in the adult is indicated.

Through a study performed on pediatric patients, subjected to nuclear-medical investigations, the validity of the LDRs is reported according to the scheme in tab. 6 (EANM-EUROPEAN ASSOCIATION OF NUCLEAR MEDICINE).

Through a study conducted in pediatric patients, subjected to medical - nuclear investigations, the importance of the dose is noted in order to obtain greater benefits in the patient and high quality images for diagnostic purposes. By carrying out pediatric-radiopharmaceutical calculations, it is possible to reproduce the aforementioned, ie an optimization of the dose.

\author{
Using body surface area \\ Child's body surface area ( $\mathrm{m} 2)$ \\ Adult's body surface area (m2) \\ $=0.53 g: 1.8 \mathrm{~g}=29 \%-2.9 \mathrm{mCi}$
}

Using organ weight

Target organ weight of child $(\mathrm{g})=93 \mathrm{~g}: 310 \mathrm{~g}=30 \%-3.0 \mathrm{mCi}$

Target organ weight of adult $(g)$

\section{INTRODUCTION}

Nuclear Medicine is of particular importance in the pediatric field; the execution of the scintigraphy allows a diagnostic approach in numerous pathologies of the child, providing indications of a morpho-functional nature of the various organs examined.

The scintigraphic examinations mainly used in pediatrics are:

- Bone scan;

- Renal scintigraphy with different tracers, such as Hippuran, MAG3 and DTPA;

- $\quad$ Renal scintigraphy with Furosemide diuretic test;

- $\quad$ renal scintigraphy with Captopril test for patients with arterial hypertension;

- Renal scintigraphy for the evaluation of glomerular filtrate;

- $\quad$ Renal scintigraphy with DMSA;

- Scintigraphy for the investigation of the ectopic gastric mucosa in Meckel's diverticulum;

- Lung ventilatory and perfusion scan;

- $\quad$ Scintigraphy with MIBG;

- Thyroid scintigraphy.

The substantial difference between the scintigraphies performed in adults and those performed in children is mainly dosimetric; it is precisely the LDRs, or the diagnostic reference levels, which regulate this aspect. The calculation for the use of radiopharmaceuticals for pediatric scintigraphic examinations and the relative tables are contained in the Legislative Decree of 26 May 2000 n. 187.

\section{NUCLEAR MEDICINE}

Nuclear medicine is the complex of knowledge, research and techniques (experimental, diagnostic and therapeutic) concerning the use of nuclear energy, and in particular of radioisotopes, in biological-medical investigations. Within these, the possibility of using radionuclides is made possible and profitable by the same behavior of living matter, whose chemical reactions are conditioned by the electronic configuration of the substances with which it comes into contact and not by their nuclear properties. It follows that the administration of stable and radioactive isotopes, used as such or incorporated in organic compounds (labeled or labeled substances), allows to induce, in radicals, molecules and even cellular elements of the organism,Although it makes use of ionizing radiation as the radiographic technique, nuclear medicine has substantial differences compared to radiology. While in X-ray techniques the localization of both the source and the sensor is known, in radiation emission techniques only the position of the sensor is known: a collimator is therefore needed to obtain an image of the space-time distribution of radioactivity in the patient.

Another important difference with respect to the radiographic technique is that while the X-ray radiation, concentrated on the structures of interest, has the duration determined by the examination modalities, the radiation of the radioactive compounds, which diffuse into the patient's body, has a duration dependent on their half-life and their escape time (1).

Radioactivity can be introduced by injection, inhalation 
or ingestion. It is therefore the patient who emits the radiations (" $\gamma$ " or " $X$ ") which are recorded by equipment that recreates the corresponding image.

\subsection{Origin}

Nuclear medicine developed in the years that followedsecond World War, representing, in a first phase, a line of research cultivated above all in the field of medical radiology but nevertheless widely applied to biochemical studies and therapy, as well as to diagnostics. The progressive deepening of the methods of investigation, the use of an ever more perfected instrumentation and the need for very special protective equipment have revealed the need for a relatively autonomous development of medicine n., Which has become the subject of university teaching. and specialization. Pediatric nuclear medicine had its clinical origins in 1946.

In particular, between 1946 and 1948, more than 1000 radioisotope thyroid studies in children and adolescents were performed in 21 hospitals in the United States. So, soon after, many doctors recognized the usefulness of nuclear medicine in pediatric patients. Among the researchers, the first was Hamilton, at the University of California, who studied a child's thyroid using I131. The development of pediatric applications was hampered over the next decade as commercially available radioisotopes and equipment were poorly suited for use in children. I131 and Hg197, for example, have high energies and a long half-life.

Well-informed people at the time were also increasingly concerned about the sensitivity of tissue development to the effects of radiation and the possibility of consequences over the long lifespan of children. The result was a rather conservative approach that limited the use of radioisotopes in the pediatric population.

Subsequently, two notable events accelerated the development of pediatric nuclear medicine. The first was the invention of the gamma camera by Hal Anger, who developed his prototype at Donner Laboratory in California between 1952 and 1958. The gamma camera is particularly suitable for pediatric imaging due to its dynamics and field of view capacity at 8 inches is, in fact, large enough to encompass most organs and the baby. The images can be obtained from a few seconds to minutes and in any case no more than an hour with the linear scanner. The gamma camera allows, in fact, the acquisition of dynamic renal function, the greatest impetus for the use of radioisotopes in children.

In the late 1960s, Nuclear-Chicago modified the electronics of their 19-inch Pho Gamma III to "split the crystal" so that both kidneys could be viewed simultaneously and radioactivity from each kidney could be recorded separately. on a chart recorder.

The second major development in the early 1960s was the clinical introduction of pertechnetate Tc $99 \mathrm{~m}$ by surgeon Paul Harper and his colleagues at the University of Chicago Hospital. The emission of an energy of 140 $\mathrm{KeV}$ of the Tc $99 \mathrm{~m}$ allowed the penetration of most of the organs of the body. The efficient energy absorbed in the thin crystal of the gamma camera increased the resolution of the image, and the short effective half-life of the radioisotope allowed for use by children. Thus Tc99m quickly replaced Hg197, which until then was the most widely used radiopharmaceutical.

From the 1960s and 1970s it was clear that the nuclear medicine techniques shown allowed for a number of applications. What was not immediately clear was how these techniques should be applied in children.
Perhaps the most fundamental principle of all pediatric practice is that children are not just miniature adults, with dosages and treatments titled by parameters of size and weight. This is especially true in nuclear medicine, where the potential effects of radioisotopes add a separate dimension to concerns about specialized approaches in children.

\subsection{Fields of application}

Particularly fruitful of results was the use of marked substances which, administered by various routes (oral, injection, inhalation), among other things allowed: a) to provide correct quantitative assessments regarding the composition of the living organism (volume total circulating blood, amount of muscle mass, etc.); b) to know the lifespan of certain cellular elements (including the corpuscular elements of the blood); c) to set up precise methods aimed at studying the functional activity of many organs (e.g., radioiodine uptake tests to evaluate thyroid function; the study of oxygen-carbon dioxide exchanges in the lungs and various tissues, etc.); d) to clarify or interpret numerous metabolic events (such as the endogenous synthesis of uric acid and cholesterol, metabolism of calcium, lipids, etc.); e) to draw diagnostic indications on the location and extent of anatomical alterations affecting various organs (see scintigraphy) and, sometimes, to provide significant guidelines on the structure of such alterations (such as the distinction of thyroid nodules in "hot" and "cold": v. thyroid); f) to facilitate the differential diagnosis between related morbid forms; g) to treat endocrine (Graves' disease) or proliferative (Vaquez's disease) or tumor diseases, exploiting the tropism of certain radioisotopes towards particular organs. to provide significant guidelines on the structure of such alterations (such as the distinction of thyroid nodules into "hot" and "cold": see thyroid); f) to facilitate the differential diagnosis between related morbid forms; g) to treat endocrine (Graves' disease) or proliferative (Vaquez's disease) or tumor diseases, exploiting the tropism of certain radioisotopes towards particular organs. to provide significant guidelines on the structure of such alterations (such as the distinction of thyroid nodules into "hot" and "cold": see thyroid); f) to facilitate the differential diagnosis between related morbid forms; g) to treat endocrine (Graves' disease) or proliferative (Vaquez's disease) or tumor diseases, exploiting the tropism of certain radioisotopes towards particular organs. As for all other medical-nuclear applications in the adult patient, pediatric nuclear medicine deals with the morpho-functional study of almost all body organs and therapy with radiopharmaceuticals; this last aspect is, with rare exceptions, limited to the treatment of some pathologies characteristic almost exclusively of the pediatric age (such as neuroblastoma) or of diseases which, although occurring more frequently in adulthood, can also affect pediatric patients (such as benign or malignant thyroid) Even if the nuclear-medical evaluation takes place in a minimally invasive way and exposes the small patient to low doses of radiation, it is nevertheless a procedure that requires diagnostic techniques in the pediatric field. The nuclear-medical investigation performed on the pediatric patient often requires changes to the standard working protocol used with adults; in particular, it is sometimes necessary to use different positions of the equipment and / or the patient during the acquisition of the exam. Even if there are no standardized behaviors and procedures (since children can be extremely different 
from each other) I have tried below to identify and describe the fundamental steps that condition the success of the pediatric scintigraphic examination. As a general warning, it is recommended to consider the medicalnuclear-pediatric approach not as an equivalent examination (carried out simply in a miniature adult), but as an investigation with its own characteristics, which must also be taken into account in the booking phase (for example, in providing for a suitable time of use for the gamma camera). Reception of the patient: it is advisable that the reception of the child and his parents be carried out by the nuclear doctor who will follow all the phases of the diagnostic examination. The nuclear doctor will introduce himself to the little patient, possibly without a gown and with colored clothes, and will try to get to know the child by communicating his or her first name. This phase must be carried out very calmly in a suitable environment (if possible, colored room with games, videos, etc.), so that the doctor can understand the attitude of the little patient and his parents. After a few minutes, the doctor must first explain to the parents what the procedures that make up the scintigraphic examination will be, inviting them to ask for any clarification they deem appropriate and trying to alleviate any anxiety in them. It is also important to involve parents in choosing the various stages of the exam (for example, any need for late acquisitions, etc.). At the end of the interview with the parents, the doctor will try to explain to the child what will happen, naturally adapting the explanation according to the age of the patient; on the basis of this interview, the doctor will be able to assess whether the child needs the presence of his parents during the successful stages of the examination or if, on the other hand, he is able to proceed alone with the examination. inviting them to ask for any clarification they deem appropriate and trying to alleviate any anxiety in them. It is also important to involve parents in choosing the various stages of the exam (for example, any need for late acquisitions, etc.). At the end of the interview with the parents, the doctor will try to explain to the child what will happen, naturally adapting the explanation according to the age of the patient; on the basis of this interview, the doctor will be able to assess whether the child needs the presence of his parents during the successful stages of the examination or if, on the other hand, he is able to proceed alone with the examination. inviting them to ask for any clarification they deem appropriate and trying to alleviate any anxiety in them. It is also important to involve parents in choosing the various stages of the exam (for example, any need for late acquisitions, etc.). At the end of the interview with the parents, the doctor will try to explain to the child what will happen, naturally adapting the explanation according to the age of the patient; on the basis of this interview, the doctor will be able to assess whether the child needs the presence of his parents during the successful stages of the examination or if, on the other hand, he is able to proceed alone with the examination. possible need for late acquisitions, etc.). At the end of the interview with the parents, the doctor will try to explain to the child what will happen, naturally adapting the explanation according to the age of the patient; on the basis of this interview, the doctor will be able to assess whether the child needs the presence of his parents during the successful stages of the examination or if, on the other hand, he is able to proceed alone with the examination. possible need for late acquisitions, etc.). At the end of the interview with the parents, the doctor will try to explain to the child what will happen, naturally adapting the explanation according to the age of the patient; on the basis of this interview, the doctor will be able to assess whether the child needs the presence of his parents during the successful stages of the examination or if, on the other hand, he is able to proceed alone with the examination.

Injection of the radiopharmaceutical: Before administration, the technician must prepare the radiopharmaceutical activity to be administered to a pediatric patient. This calculation can be based on weight or body surface area, or on predetermined values according to age brackets and so on, as long as the result is to determine the minimum activity necessary to be able to perform a satisfactory scintigraphic examination, while respecting the containment of the doses provided according to Legislative Decree 187/2000 of 26 May 2000 (LDR). I report below, as an example, the formula of the percentage activity as a function of weight: Activity to be injected = It is recommended, in this regard, to take into account the recommendations recently developed by the Dosimetry and Paediatrics Committees of the European Association of Nuclear Medicine. The injection ev of the radiopharmaceutical is certainly the worst moment for the little patient, whatever his age. His ability to self-control is often non-existent and the needle almost always remembers moments lived with pain; it is therefore necessary to (Child weight in $\mathrm{kg}$ ) $(70 \mathrm{~kg}) \times($ maximum activity in adult $)$

Diagnostic techniques in the pediatric field: before performing the injection, carefully inspect all possible access routes, in order to minimize the time required to immobilize the arm. It is certainly useful, when possible, to actively involve parents, who will be asked to distract the child during the doctor's maneuver. Patient positioning during scintigraphic acquisition: The purpose of correct positioning of the small patient is to ensure his immobility under the gamma camera to allow adequate scintigraphic acquisition. Depending on the age, various methods can be adopted: one of the most effective consists in wrapping the child with a sheet or blanket and fixing his supine or prone position with velcro strips, with a maneuver that may seem to the child almost a game and can therefore facilitate its compliance. If possible, it is preferable to leave the parent in the room with the child to give him greater security in a strange environment. In some particularly difficult cases and in case of planar acquisitions, it is advisable to have the baby lie down on the parent's body already positioned on the gamma camera bed. When the patient is unable to collaborate with the doctor and the technician, and the parents request sedation, this must be performed taking into account the advantages (it makes it possible to acquire better quality images and SPECT / CT of body regions such as head and neck) and the disadvantages (new IV injection, possible unexpected reactions for which the child becomes hyperactive ; finally, albeit infinitesimal.

The diagnostic applications of pediatric nuclear medicine concern pathologies of the skeleton, kidney, esophagus-gastric tract, heart, lung, liver, central nervous system, as well as some congenital diseases and investigations in the logical infectious field. Radioactivity is induced with radioactive materials called radiopharmaceuticals or radiotracers, which collects in the area of 


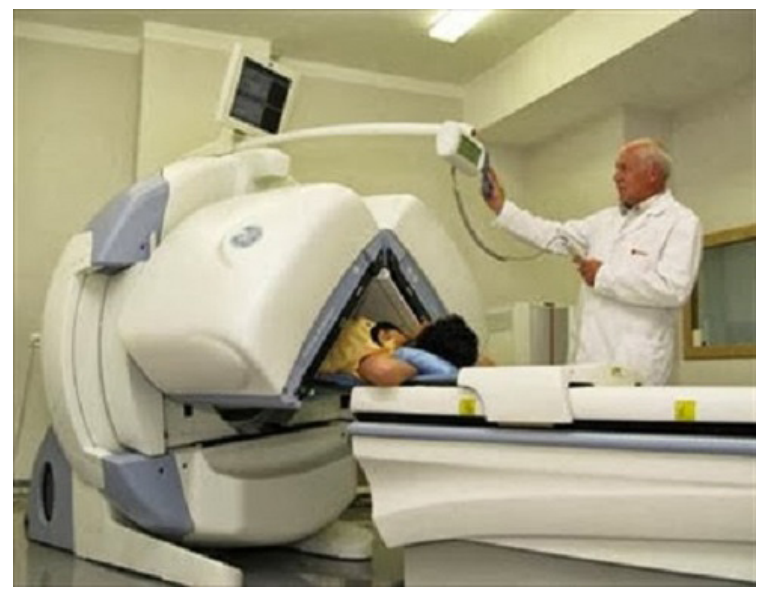

Fig. 1 - Pediatric patient scan.

the body to be examined, giving off energy in the form of gamma rays $(\gamma)$.

\subsection{RADIO DRUG}

A radiopharmaceutical is defined as "any medicine which, when ready for use, includes one or more radionuclides, incorporated for sanitary use" (EEC directive $89 / 343$, legislative decree 187). It is therefore a radioisotope-labeled compound, obtained by binding radioactive molecules emitting gamma photons or positrons to biological molecules of clinical interest.

The type of molecule chosen depends on what information is required. Isotopes are different forms of the same element that have the same atomic number $\mathrm{Z}$, but have a different mass number A; therefore they have the same number of protons (and therefore of electrons) but different number of neutrons. The isotopes of an element have the same chemical characteristics, even if they can be physically different, for example stable (ie non-radioactive) or unstable (ie radioactive). The most widely used radiopharmaceutical today is Technetium 99m which, in addition to being cheap and easily produced on site, emits only gamma radiation, of energy suitable for obtaining images and does not contaminate the environment because it has a half-life of only 6 hours and therefore runs out quickly his business.

\subsection{CAMERA RANGE}

The gamma camera is used for the acquisition of static, dynamic and tomographic scintigraphic images, expression of the in vivo distribution of appropriately administered radiopharmaceuticals.

Since it allows the simultaneous exploration of large body areas, it is today the most widely used equip-

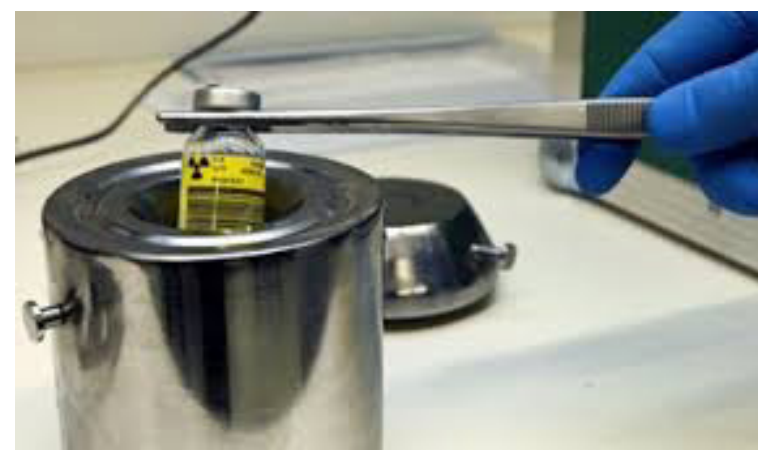

Fig. 2 - Radiopharmaceutical. ment for diagnostic investigations in nuclear medicine, where it has replaced the old linear scintigraph which, by performing a point-by-point scan, required long acquisition times. and provided only static planar images. It is also called "Anger's room" in honor of the engineer who was the inventor and builder of the first prototype in 1953 and who extensively developed the characteristics of the aforementioned equipment.

Over time it has been perfected to be best used with the $99 \mathrm{mTc} 140 \mathrm{keV}$ gamma photons which is the most widely used radionuclide in nuclear medicine diagnostics today due to its physical and dosimetric characteristics, its easy supply and low cost.

It is important to underline that, unlike radiological equipment, the gamma camera does not emit radiation but is limited to detecting the radiation emitted by the patient.

\subsection{Components}

The main components of a modern gamma camera are:

- A collimator which is placed between the object emitting the radiation and the detection crystal. It has the function of shielding the radiations directed towards unwanted directions that would degrade the quality of the scintigraphic image;

- Acrystallarge surface, circular or rectangular, for the detection of $\mathrm{x}$ and gamma radiation which are transformed into luminous photons ("sparks"). The width of the crystal determines the field of view of the gamma camera;

- Thephotomultipliers (pm) and the preamplifiers which, connected to the rear surface of the detection crystal by means of an optical connection, have the function of detecting the light photons by transforming their energy into an electrical signal which is amplified and conducted towards the processing circuits;

The processing circuits, schematically distinguishable in:

- Spatial localization circuit, which determines where the interaction of the gamma ray occurred in the crystal;

- Pulse-height analyzer, which recognizes the energy of the incident radiation and allows a selection of the pulses useful for image formation;

- Analog-to-digital converters (adcs) that transform the signals coming from the pulse processing circuits from analog to digital, thus allowing them to be inserted into the memory of a computer;

- A computer, with which the acquired data are stored and processed, making it possible to obtain the final images to be printed on radiographic film or paper supports, in color or black and white, which can be used for clinical-diagnostic purposes.

The collimator is a structure of a metal with a high atomic number and therefore with a high radiation shielding power (generally lead). It is made up of metal partitions that surround holes, mostly arranged in a "honeycomb" shape, which are crossed by radiation. It has the function of "focusing" the photonic beam before it reaches the detection crystal. 


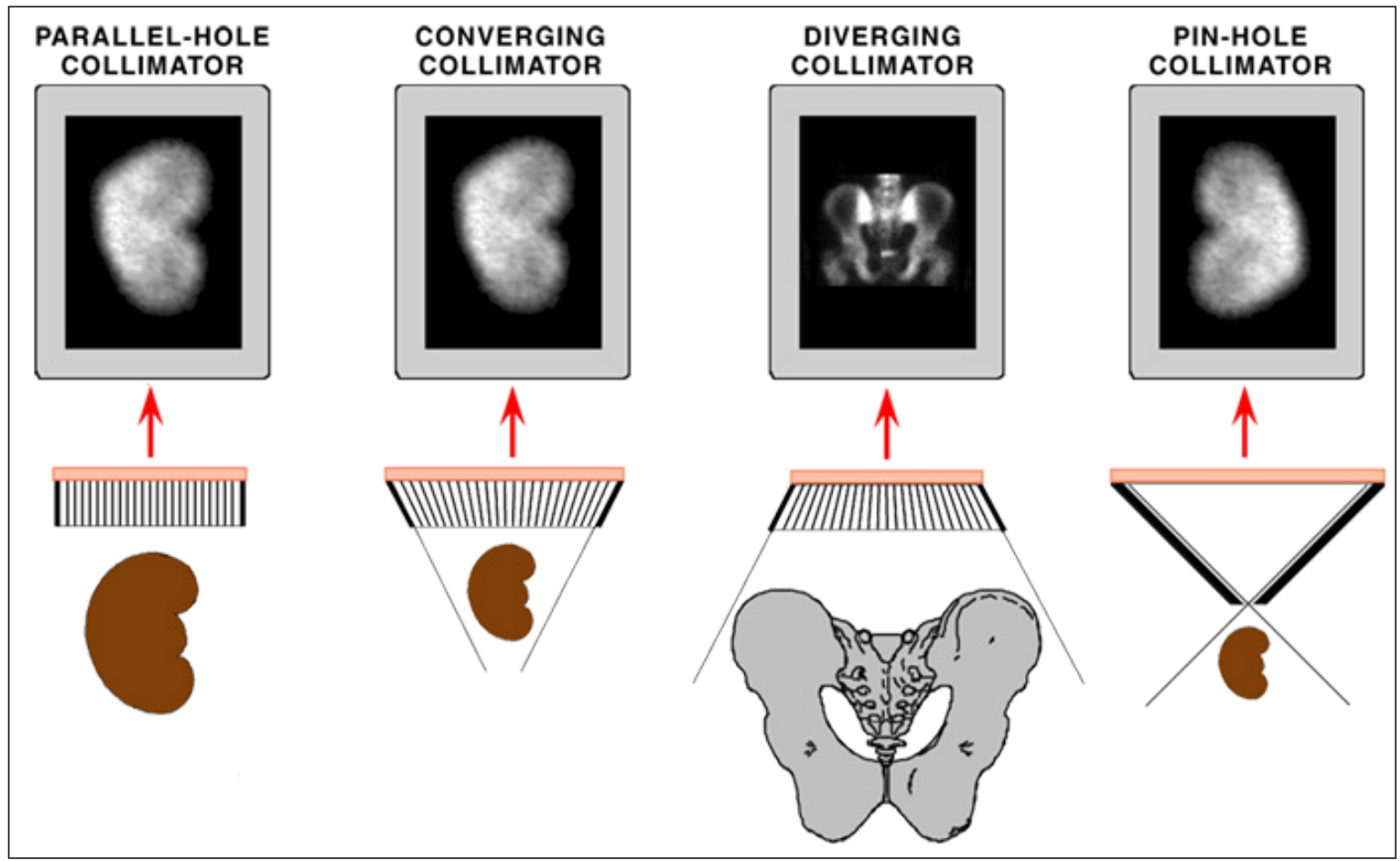

Fig. 3 - Collimators.

The focusing of the gamma rays is a focusing by absorption: From the source, located near the gamma camera, the photons come out in all directions. Those that reach the gamma camera therefore come from every area of the source in a completely random way and this would not allow to produce an informative image. The gamma photons, due to their penetrating power, cannot be deflected by optical lenses, as happens for the luminous photons in traditional photography; Furthermore, since they do not possess an electric charge, they cannot be deflected by electric or magnetic fields. The photonic beam is then modulated by means of the collimator which acts by shielding most of the photons and letting pass those whose trajectory passes through the focal point. Collimation by absorption therefore allows you to project an image on the detection crystal formed only by gamma photons that reach it from certain directions. Focusing by absorption is an intrinsically ineffective method, because it inevitably rejects most of the radiation emitted by the patient. This is one of the reasons behind the lower morphological detail expressed by nuclear-medical images compared to radiological ones.

- The thickness of the metal septa of the collimators is variable according to the energy of the photons to be focused, as the absorbing power of the septa is reduced as the energy of the incident photons increases. Therefore, to obtain a good quality image using nuclides emitting high energy radiation, heavy collimators with large septa are required, which however reduce the resolving power of the image itself and the sensitivity of the system.

According to their geometry, collimators are divided into four main types:

- $\quad$ parallel-hole- the most used, focusing at infinity, has holes parallel and perpendicular to the surface of the crystal. Projects an image of the same size and without spatial distortion of the explored structure. A variant of the same is the "slant-hole" which has parallel holes but with a fixed angle with respect to the perpendicular on the surface of the crystal (usually $25^{\circ}$ )

- $\quad$ converging- today little used, it has converging holes and can be considered an inverted diverging of which it maintains all the drawbacks. Similar to the pin-hole, it enlarges the image and has been used in the past to study small organs such as the thyroid gland or for pediatric studies.

- diverging- today little used thanks to the large size of the revelation crystals currently used. It has diverging holes that have the function of increasing the field of view of the crystal and therefore of visualizing large organs or large body surfaces on crystals smaller than them. It was widely used in the past due to the small size of the crystals of the old gamma cameras. It has the defect that the magnification factor and the spatial resolution of the image obtained are variable depending on whether the structure explored is located near the center of the crystal or in the periphery, in superficial or deep planes. This introduces a distortion of the image which can cause difficulties in interpreting the image.

- $\quad$ pin-hole- focusing at a short distance, enlarges the image. On the other hand, it introduces a distortion due to the different distance from the focus of the explored structures and provides an inverted image on both axes. It is used for small organs, such as the thyroid gland, or to obtain enlargements of small parts of the body.

The detector crystal is a scintillation detector, usually sodium iodide (NaI) activated with thallium (Tl). The $\mathrm{NaI}$ crystal acquires its sparkling properties thanks to the presence of the $\mathrm{Tl}$ impurities inserted in the crystal lattice. These impurities act as electron traps and form luminescence centers. The crystal is very delicate and must be protected from humidity which drastically degrades its qualities. For this reason it is enclosed in an aluminum layer, internally coated with reflective ma- 


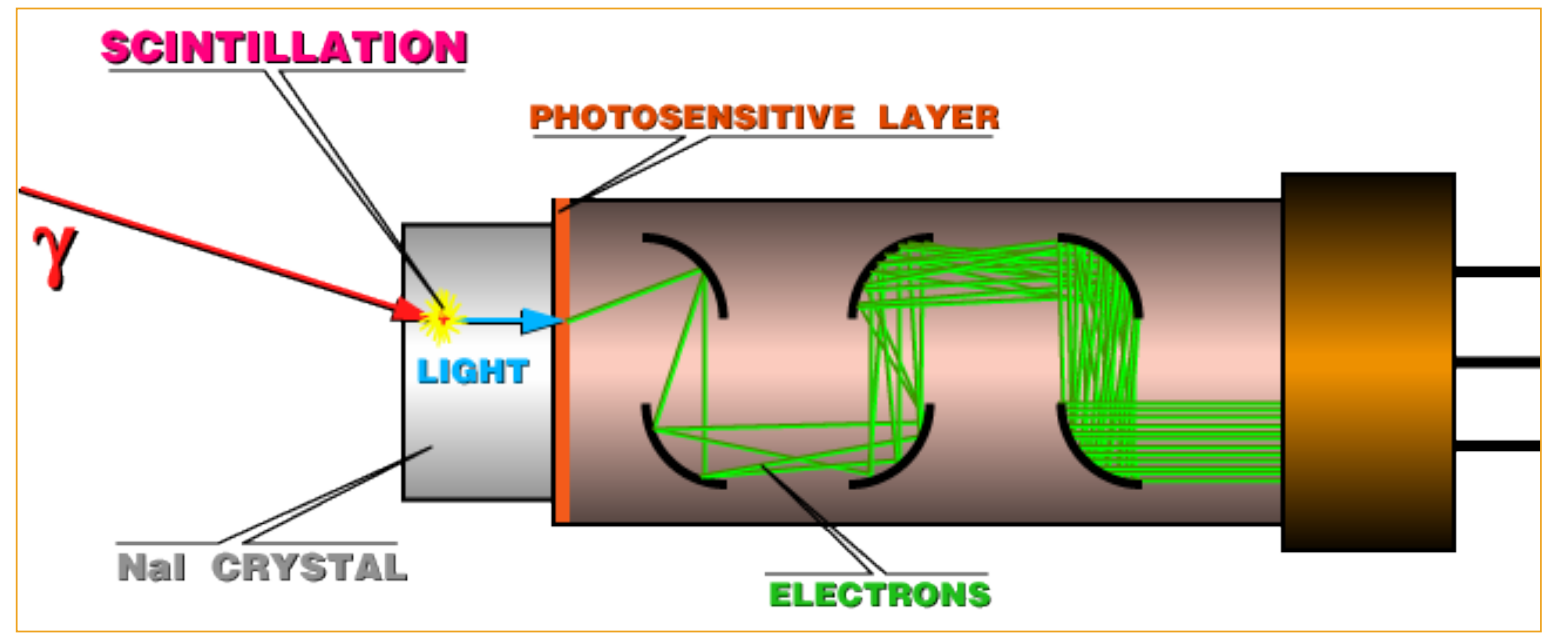

Fig. 4 - photomultiplier.

terial (light guide) except on the rear side where it is coupled to the photomultipliers.

When the gamma photon enters the crystal it interacts with it through a series of Compton collisions in rapid sequence, dissipating the residual energy in a photoelectric interaction. Not all the ranges that reach the crystal, however, interact with it, some cross it: for energies of the range below $200 \mathrm{keV}$ the efficiency of the crystal is about $80 \%$, while for higher energies the efficiency is drastically reduced. Whenever a Compton collision or photoelectric interaction occurs, the energy of the gamma is partially or totally transmitted to an electron which, moving in the crystal lattice, reaches a center of luminescence where flashes of light are produced, in the length of wave from blue to ultraviolet. Although emitted in sequence, the flashes of light deriving from the interaction with the crystal of the same gamma photon reach the photomultiplier in a time shorter than the possibility of temporal discrimination which sees them as a single luminous flash, of intensity proportional to the energy of the incident gamma photon (typically 30 bright photons). Thus, if the incident photon dissipates all its energy in the crystal, a perfect proportionality is maintained between the energy of the gamma photon and the intensity of the light reaching the photomultipliers. This is of fundamental importance for the subsequent stages of signal processing. if the incident photon dissipates all its energy in the crystal, a perfect proportionality is maintained between the energy of the gamma photon and the intensity of the light reaching the photomultipliers. This is of fundamental importance for the subsequent stages of signal processing. if the incident photon dissipates all its energy in the crystal, a perfect proportionality is maintained between the energy of the gamma photon and the intensity of the light reaching the photomultipliers. This is of fundamental importance for the subsequent stages of signal processing.

Modern gamma cameras are generally equipped with circular crystals, with a diameter of $40-50 \mathrm{~cm}$, or rectangular with sides of $30-40 \times 40-50 \mathrm{~cm}$.

The photomultipliers coupled in close contiguity with the back of the crystal consist of a photocathode and a chain of dinodes.

- In the photocathode the flash of light is converted by photoemission into electrons while maintaining the proportionality between light energy and the number of electrons torn from the photocathode.

- In the dinodes, positioned in series and maintained at a high and constant potential difference by means of a power supply, the signal is amplified: the electrons arriving at the first dinode of the PM are accelerated by the electric field towards the second dinode where they collide producing the secondary emission of other electrons which in turn are accelerated towards the third dinode and so on. In this way a cascade multiplication of the electrons is produced which, at the output, are treated as a pulse of electric current. The proportionality between the intensity of the light pulse and the amplitude of the electrical signal is respected.

The gamma rays emitted by the patient, after passing through the collimator, are converted into sparks of light in the detection crystal. This creates a virtual image of scintillation inside the crystal, which reflects the distribution of radioactivity in the patient.

- The spark of light is "seen" by each photomultiplier with an intensity inversely proportional to its distance from the point where the scintillation took place in the crystal. By photoemission, light is converted into electrons. This small electrical signal is amplified by the photomultiplier and processed by special circuits capable of locating the origin of the event. In practice, since the amplitude of the output signal from the PM is inversely proportional to the distance between the center of the photocathode and the point of the crystal where the scintillation was produced, the distribution of the amplitude of the output signals from the various PM allows the circuit location to provide the $\mathrm{X}$ and $\mathrm{Y}$ coordinates of the pulse.

- The output signals from all the contiguous PMs are added together to produce a pulse called $\mathrm{Z}$ which has an amplitude directly proportional to the intensity of the light produced in the crystal and therefore directly proportional to the energy of the incident gamma ray. An analysis circuit of the amplitude of the signal $\mathrm{Z}$ allows to reject all the signals that are not within an energy "window" chosen by the operator. In this way it is possible to eliminate part of the "scatter" radiation (Compton effect), which having diverted their direction after being emitted produce a degradation of the 


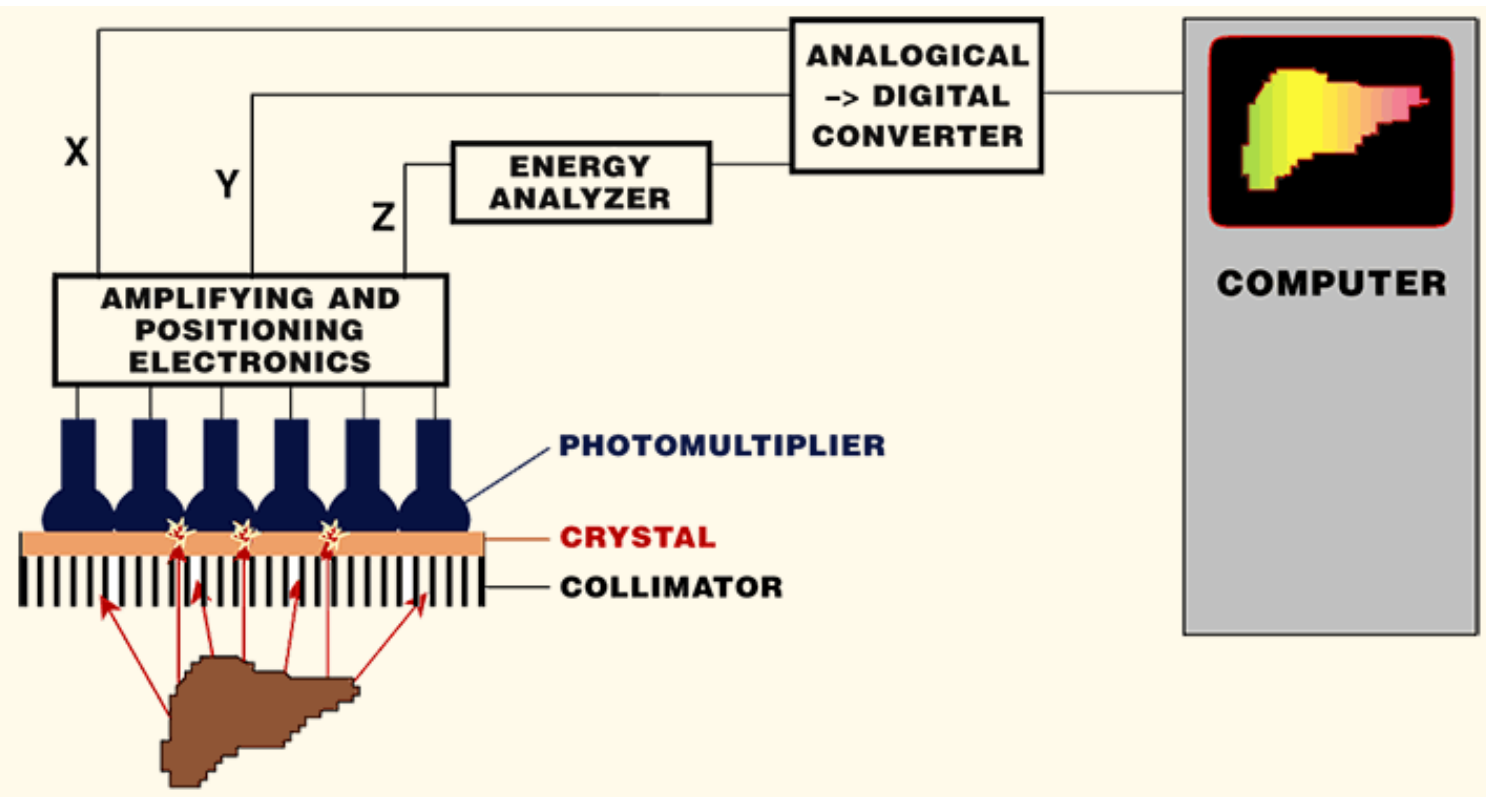

Fig. 5 - path of the radiation.

image. The ability to recognize the energy of the photon that causes the scintillation also allows the simultaneous use of several radiopharmaceuticals that emit photons of different energy. By administering at the same time to the patient, for example, 99mTecnezio (140 keV) and 201Tallio $(70-80 \mathrm{keV})$ it is possible to program the equipment so that the acquired signals are recorded on two different images, corresponding respectively to $99 \mathrm{mTc}$ and $201 \mathrm{Tl}$.

- $\quad$ Finally, the signals are digitized (ie converted from simple electrical signals to numbers) so as to allow them to be stored in a computer, in the form of numerical matrices, which can then be processed in the ways deemed appropriate.

\section{Digital scintigraphic images}

- In modern gamma cameras, scintigraphic images are usually acquired digitally, in the form of twodimensional numerical matrices (square or rectangular) formed by a set of elements called pixels (from the English "picture element"). In the computer memory, each pixel can correspond to one or two "bytes", ie a binary number of 8 or 16 bits respectively. In the first case the maximum count that can be recorded in a pixel is therefore 255 (equal to $2 \wedge 8-1$ ) in the second case it is 65535 (equal to $2^{\wedge} 16-1$ ). The number of photons that the system, during data acquisition, identifies as coming from the corresponding small portion of the object being examined is recorded in each pixel. It is therefore evident that the final resolution of the image will also depend on the size of the chosen matrix. The larger the matrix, the smaller the pixels that compose it (with the same dimensions of the explored field) and therefore the better the resolution of the final image (meaning as resolution the shortest distance at which two neighboring points can be correctly identified as distinct).

- In nuclear medicine the matrices usually used are $64 \times 64,128 \times 128,256 \times 256$ and $512 \times 512$ pixels. In fact, if we consider, by way of example, that the image is acquired with a gamma camera with a field of view of $40 \times 40 \mathrm{~cm}$, a pixel will correspond respectively to a portion equal to $6.2,3.1,1.5,0.75$ $\mathrm{mm}$. As a general rule of image digitization establishes that the optimal matrix to be used is the one formed by pixels having half the maximum resolution obtainable by the image acquisition system as size and considering that at the current state of technology the gamma cameras do not are able to provide images with a spatial resolution better than 3-4 $\mathrm{mm}$, it would be unnecessarily expensive to use matrices larger than $512 \times 512$ pixels.

- To recreate the image on the monitor, the computer assigns to the numerical value contained in each pixel a color level determined on the basis of a color table arbitrarily chosen by the operator. The most common, and generally more effective, solution is to use a gray scale in which the highest number of counts corresponds to white and the lowest to black; the intermediate counts will be represented by the various gray levels.

\subsection{THE SCINTIGRAPHIC EXAMINATION}

With common X-ray examinations, an image is made by passing X-rays through the body out of the source. In contrast, nuclear medical procedures use a radioactive material called a radiotracer, which is injected into the blood, swallowed by mouth, or inhaled as a gas.

The radioactive material accumulates in the organ or in an area of the body to be examined, from which a small amount of energy in the form of $\gamma$ rays emanates.

A gamma camera, PER tomograph or probe detect this energy and with the help of a computer create images that offer detailed information on both the structure and function of organs and other parts of the body.

\subsection{The examination execution}

When necessary, the nurse inserts a cannula needle into the vein in the arm or hand.

Depending on the type of nuclear medical examination you are undergoing, the radiotracer dose can be injected intravenously or swallowed by mouth. 
It takes a few seconds to a few days for the radiotracer to pass through the body and accumulate in the organ or area to be studied. Consequently, the acquisition can be done immediately, a few hours later, or even a few days after receiving the radiopharmaceutical.

When it is time to begin the acquisition, it is necessary to lie down on the bed. The gamma camera will then make a series of images rotating around the patient (SPECT) or standing still in a single position and the patient will be asked to change position between acquisitions. While the gamma camera is acquiring, the patient will need to remain still for short periods of time.

The actual scan time ranges from 20 minutes to several hours.

When the exam is complete, the patient may be asked to wait for the technician to check the images to see if further images are needed.

After the examination, if the patient has a cannula needle, this will be removed.

\subsection{ADVANTAGES}

\section{AND DISADVANTAGES}

In nuclear medicine special radioactive tracers are used which follow the path of the molecules present in the body and which can be detected from the outside. In this way we are able to know in depth the path and also the concentration of a given molecule in the different parts of the body. This is a huge advantage for doctors, since it allows you to have detailed information both on the diseased areas and on the functionality of the various organs on each individual patient. The term "nuclear" is now having to deal with the prejudices linked to the disasters of Fukushima or Chernobyl, however the risk of undergoing a nuclear medicine exam is minimal and comparable to the risks present in everyday life. We need to know that radiation is commonly found in the atmosphere and an airplane pilot absorbs far more radiation in a month than any of our patients.

Furthermore, to perform a diagnostic test, the patient is administered micro-quantities of radioactive substances: also for this reason, nuclear medicine tests today have no absolute contraindication, as opposed to what happens with magnetic resonance or $\mathrm{CT}$, in which there is no all subjects can be studied and contrast agents are used which can, although fortunately very rarely, determine the patient's death. Given the general rule that all radiological investigations should be requested and performed only when it is assumed that they are able to provide useful information for the patient [but this rule should be applied for all diagnostic procedures] there is only one absolute contraindication: pregnancy, and a relative one: breastfeeding. In the event of an ascertained pregnancy, no radiological investigation should be performed, and therefore also nuclear medicine, unless the obtainable information is considered essential for the patient's life. That is, the benefits must outweigh the potential risks to the unborn child.

Regardless of these regulations, which are the result of precise legislative provisions, the dose of radiation absorbed by the embryo or fetus for the possible execution of a scintigraphic investigation in the event of an unrecognized pregnancy is in any case very low and significantly lower than the levels who might recommend a therapeutic abortion.
As for breastfeeding, it is almost always sufficient to suspend it for a time that varies according to the radiopharmaceutical used but which, in most cases, does not exceed 24 hours.

5.1 Limitations of the nuclear medical examination As mentioned above, nuclear medical procedures can take time, they can take hours to day for the radiotracer to accumulate in the part of the body to be studied and the acquisition can take up to several hours to perform, although the new equipment available significantly reduces the procedure time.

The resolution of body structures with nuclear medicine may not be as clear as other imaging techniques, such as CT or MRI. However, the information gained from nuclear medicine is unequal in other imaging techniques.

\section{NUCLEAR MEDICINE}

\section{IN PEDIATRICS}

In children, nuclear medicine finds an ideal field of application, because it provides, in a non-invasive way and with a low exposure to ionizing radiation, precisely those functional information that are essential to prevent the onset of damage in the developing organism. . Surely the most frequent application concerns the study of dilations of the urinary tract (hydronephrosis) which today are almost always discovered already during pregnancy or, at the latest, at birth.

Many of these cases recover spontaneously without the need for surgical treatment, which is more often reserved for cases where renal function assessed by scintigraphy is threatened.

Another typical case is the study of kidney infections (pyelonephritis) both for diagnosis and to check if the infection has led to permanent tissue damage (kidney scarring).

In all these cases the method has the great advantage of being perfectly tolerated even by the kidneys of a newborn, even during an infection, even in premature babies.

Nuclear medicine also provides very useful data in the study of some congenital heart disease, for the study of blood flow in the lungs or in the heart muscle. It is possible to perform in Pediatricsalmost all the scintigraphic investigations foreseen for the adult, the methods of execution are modified, in order to be able to adapt to the particular clinical situations and to the behavioral characteristics typical of pathologies of the pediatric age.

\section{${ }^{6}$ PEDIATRIC SPARI - TECHNICAL ASPECTS"}

\section{PEDIATRIC BONE} SCINTIGRAPHY

Bone is a dynamic system sensitive to external mechanical stimuli subject to constant internal remodeling, consisting of resorption and formation of new tissue.

Three types of cells are involved in the genesis and resorption of bone: ${ }^{-}$osteoblasts, which produce the organic matrix;

- osteocytes, which synthesize the inorganic matrix and release it to the outside by exocytosis;

- $\quad$ osteoclasts, which activate bone resorption.

There are 2 types of ossification:

- endochondral: replacement of cartilage with 
bone in the centers of ossification (limbs, spine, ribs, skull base)

- intramembranous: osteoblastic differentiated mesenchymal cells produce the organic matrix in which osteocytes synthesize the inorganic matrix. It is the type of ossification that affects the cranial vault, the maxilla and the mandible.

About $65 \%$ of bone is composed of a mineral phase formed by hydroxyapatite crystals $\mathrm{Ca} 10(\mathrm{PO} 4) 6(\mathrm{OH})$ 2 and sodium, magnesium, carbonate and citrate ions, and for the remaining $35 \%$ by an organic phase consisting of $95 \%$ from collagen fibers and $5 \%$ from mucopolysaccharides

\subsection{Physiopathology of the bone}

It exploits the characteristic of some radiopharmaceuticals to concentrate in the bone in a proportional way to the vascularization and osteoblastic activity, thus allowing to evaluate the degree of metabolic activity of the skeleton and to recognize any lesion that causes a district alteration of the metabolic turnover of the bone. It allows the simultaneous exploration of all skeletal areas, in a short time, with low irradiation and with high diagnostic sensitivity.

Other non-negligible advantages are the simplicity of execution, non-invasiveness and low cost.

\subsection{General}

Bone scintigraphy allows to evaluate the physiology of the bone and the anatomy of the skeleton and represents one of the most common applications of Nuclear Medicine

- provides physiological information relating to bone perfusion and metabolism

- allows the study of the entire skeletal system as it provides the map of the distribution of the radiopharmaceutical throughout the body, highlighting the degree of osteoblastic activity of the various skeletal areas

- it is a highly sensitive method. It has a high diagnostic sensitivity since it allows to recognize alterations in the district bone metabolism before they become radiologically detectable.

- For a bone lesion to be highlighted with a traditional radiological examination, a reduction of the calcium content of more than $35-40 \%$ is necessary, while a modest variation (approx. $2 \%$ ) is sufficient for it to be detectable with a scintigraphic examination.

- is characterized by a rather limited specificity, since any pathology capable of inducing an increase in osteoblastic activity causes hyperconcentration of the radiopharmaceutical.

- a specific diagnosis can often be obtained when the scintigraphy is correlated with radiological examinations or other imaging methods

- Technetium-99m-labeled diphosphonates are the tracers of choice for bone scan. They passively diffuse into the extravascular and extracellular spaces and are deposited around the bone matrix. The unbound portion of the tracer is eliminated from the plasma by renal excretion. examination is performed 2-5 hours after injection to obtain a high bone / fundus ratio, which guarantees good image quality. The greater the blood flow and metabolic activity of a particular bone region, the greater the concentration of the tracer.
High quality images are important and require immobilization and correct positioning of the child as well as optimal equipment. Two- or three-stage bone scans are the most commonly used.

This technique has a high sensitivity in the early diagnosis of metabolic bone pathologies by detecting bone diseases. Traditional X-ray techniques, which describe morphological changes, are less sensitive at the onset of a disease process. A negative bone scan usually rules out significant bone disorders with a high degree of certainty. this examination technique does not provide information regarding fractures. It is, in fact, a "nonspecific" examination since, both in the case of metastases and fractures, we see an area of hyperaccumulation. Wherever there is a bone lesion, the osteoblasts (bone cells that participate directly in the formation of the bone by secreting the organic compounds of the matrix and regulating the deposition of mineral salts) capture the tracer administered to the patient.

\subsection{Indications in carrying out the exam}

Bone scan is indicated whenever there is a suspicion of skeletal pathology.

The main clinical indications are:

- TO Inflammation or infection

- Acute osteomyelitis against soft tissue inflammation

- Subacute and chronic osteomyelitis

- Aseptic arthritis.

- B. Bone tumors:

$\circ$ Benign bone tumors

- Malignant bone tumors

- Bone metastases

- C. Aseptic necrosis

- Legg-Calve-Perthes disease

- Aseptic necrosis other than Legg-CalvePerthes disease e bone infarction

- Sickle cell disease

- D. Traumatic bone diseases

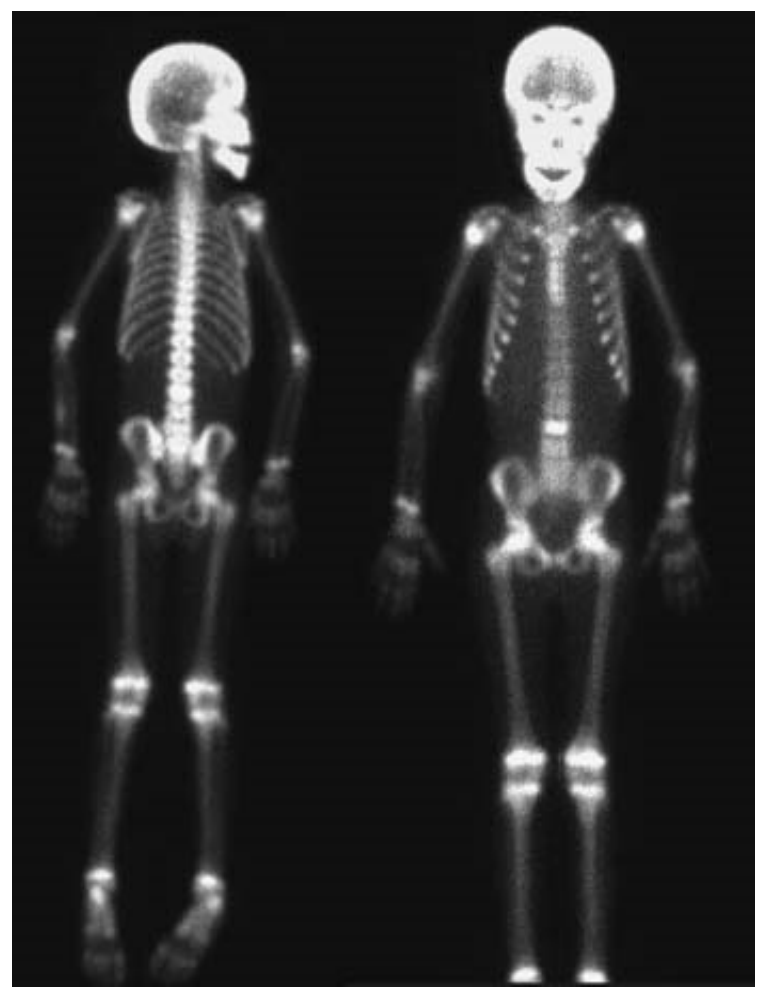

Fig. 6 - Example scintigraphy. 
Equivocal X-ray results after trauma Forced fractures

- Child abuse (battered child syndrome)

- Politrauma

- Complications of fractures and therapy

- A Sudeck's atrophy - Sympathetic reflex dystrophy

- B Surgically guided bone scan

- C Bone dysplasia

○ For example, Camurati-Engelmann disease

- D Other clinical situations in pediatrics

- Pain possibly due to bone disease

- Child with limp or back pain

- Child who refuses to stand up or use one of the limbs

- Fever of unknown origin

\subsection{Radio drug used}

7.4.1 Radionuclide

The radionuclide used is Technetium-99m (Tc99m)

\subsubsection{Drug}

The drugs used for this type of investigation are polyphosphates such as:

MDP (methylenediphosphonate)

HMDP or HDP (hydroxymethylenediphosphonate)

DPD (diphosphonopropanedicarboxylic acid)

\subsubsection{Dosage}

Minimum dose: $40 \mathrm{MBq}$

Maximum recommended dose: $500 \mathrm{MBq}$

The administered dose should be spread over a basic body surface area.

\subsubsection{Burden of radiation}

The radiation burden depends on the age of the child.

For an infant receiving Tc99m, the effective equivalent dose is $0.11 \mathrm{mSv} / \mathrm{MBq}$. It decreases with age $(0.042$ $\mathrm{mSv} / \mathrm{MBq}$ - 1 year of age; $0.021 \mathrm{mSv} / \mathrm{MBq}$ - 5 years; $0.014 \mathrm{mSv} / \mathrm{MBq}-10$ years and $0.0089 \mathrm{mSv} / \mathrm{MBq}$ - 15 years).

The radiation dose estimate is higher for the bone surfaces (1.6 mGy / MBq in a newborn) and for the bladder wall (0.4 mGy / MBq in a newborn). This decreases to values of $0.0076 \mathrm{mGy} / \mathrm{MBq}$ (bone surface) and $0.042 \mathrm{mGy} / \mathrm{MBq}$ (bladder wall) in a 15-year-old child.

The radiation burden is calculated based on the assumption of bladder emptying within 3 hours. If oral fluid intake is stimulated resulting in faster bladder emptying, then the radiation dose will be reduced.

\subsection{Execution of the examination}

Patient position: supine.

For the three-phase study technique, the computer is started simultaneously with the injection of the tracer. In the one-step study technique, the injection can be done near the gamma camera or even in another room.

\subsubsection{Acquisition of images}

A total body bone scan is usually performed which consists in the acquisition of images of the entire skeletal system by means of a scan of the patient in the anterior and posterior projections, after 2-3 hours from the injection.

\subsection{Three-phase spark}

It is performed on single skeletal areas where suspected pathology is located, including, when possible, also contralateral skeletal portions or segments in the field of view. Patient positioning and the projection used in the study must remain constant. It consists of three phases: angiography; blood-pool; static. It is followed by a whole-body acquisition

- Angiographic phase (first): 128 x 128 or 256 x 256 matrix. Dynamic acquisition starting at the same time as the injection ev.

- $\quad$ Scanning speed: 2 seconds / image for 60 images

- Blood-pool phase (second): $128 \times 128$ or $256 \times$ 256 matrix, dynamic acquisition starting at the end of the first phase and continuing up to 10 minutes after administration. The images are obtained 30 seconds / image or static acquisition (5001000Kcounts / projection) for a total of 6-10 images

- Metabolic or late phase (third): 256 x 256 matrix, static acquisition three to four hours after administration

- Whole-body scan: front and rear projection body scan with 256 x 1024 matrix with $12-15 \mathrm{~cm} / \mathrm{min}-$ ute scanning speed with at least 1500 kcounts / projection

\subsubsection{Analysis of the images}

Qualitative analysis

consists in the visual examination of the images in search of areas of increased fixation (hyperfixation) of the radiopharmaceutical in correspondence of the skeletal segments, or of an entire segment or of a single part of it (diffuse or focal hyperfixation).

- Areas of reduced or absent skeletal fixation should also be described.

- the analysis must include: skeletal segments, joints, muscle tendon insertions.

Quantitative analysis

It consists in the comparison of the counts contained in regions of interest (ROI) drawn on symmetrical skeletal districts or on adjacent bone elements.

Normal wholebody bone scan shows the axial and appendicular bone skeleton

In the young subject, before the completion of skeletal growth, the growth plates are the site of physiological increased fixation of the radiopharmaceutical

- the increase or decrease in skeletal fixation is always defined in relation to that of normal bone and is the expression of an increased or reduced osteoblastic activity

- most bone lesions manifest as an area of increased fixation of the radiopharmaceutical compared to fixation of the corresponding contralateral skeletal segment or the closest (over or underneath) normal bone structures

- $\quad$ Kidney visualization is normal if no more than 4 hours have passed since the injection.

- In pediatric patients, the growth plates appear clearly hyperactive and must be well delimited and symmetrical; cranial sutures are also commonly seen.

\section{RENAL PEDIATRIC SCINTIGRAPHY}

The kidneys are two organs that produce and secrete urine. In this way they intervene in the regulation of the 
volume and composition of body fluids. Mainly they perform four functions:

- They participate in the regulation of the hydroelectrolytic balance through the secretion of water and electrolytes. In this way, internal production and external input are balanced.

- They participate in the regulation of the production, absorption and excretion of acids and bases.

- They eliminate some metabolic products, no longer needed by the body (urea, uric acid, creatinine, etc.) which, if not eliminated, are toxic for the body.

- They produce some hormones necessary for the regulation of blood pressure and blood circulation (prostaglandins, angiotensin, renin), in the production of red blood cells (erythropoietin) and in the regulation of phosphorus and calcium metabolism (calcitriol).

The kidneys, equal organs retroperitoneal. In the section of a kidney the following portions can be observed: the cortex, which is the peripheral area containing the nephrons, the medulla, which is the intermediate portion and contains the urine collector ducts, and the sinus renal which represents the seat of the renal pelvis and the entrance to the vessels. The functional unit of the kidney is the nephron which is responsible for filtering the various blood solutes and producing urine. The urine is poured into a collection system which is the collecting system of the kidney: the calyxes and the renal pelvis, which continues with the ureter, a tube that for the final transport of urine into the bladder.

From a structural point of view, therefore, the kidney is made up of two different portions: the renal parenchyma, responsible for producing urine, and the collecting system or renal cavities, which are part of the urinary or excretory pathway. This distinction is important because renal parenchyma cells are different from urinary tract cells. The cells of the kidney cavities are the same cells that make up the mucosa (the layer that covers the inside) of the ureter and bladder.

The kidneys can be affected by various morbid processes, of both nephrological and urological interest. Inflammatory processes of the kidney (glomerulonephritis, pyelonephritis, tubulo-interstitial pathologies) are of nephrological interest. The pathologies of urological interest are represented by stones, tumors of the renal parenchyma or excretory tracts, renal malformations.

\subsection{General}

The scintigraphic imaging, without invasiveness and with a modest dose to the patient, allows the pathophysiological study of the kidney and urinary tract It can be repeated over time as functional monitoring (important for pediatric patients). The use of pharmacological tests (ACE inhibitor,

diuretic) allows to evaluate some pathologies otherwise difficult to diagnose non-invasively.

The main role of renal scintigraphy in Pediatrics is the evaluation of the existence and location of the kidneys (hypo-aplasia, ptosis, agenesis, etc.).

Dynamic scintigraphy allows the estimation of two aspects of renal function (5).

- The first aspect is renal clearance (volume of plasma from which the kidneys are able to eliminate a certain substance in the unit of time);
- The second function, which can be evaluated on the basis of renography, is the excretion of the tracer from the kidney. This disappearance cansimply be estimated by the renogram check: an early peak followed by a rapid descending phase is typical for normal secretion. An important delay in excretion is characterized by a continuously rising curve.

To quantify the transit of tracer in the kidney, various techniques have been proposed ranging from simple descriptive parameters (such as the time to reach the maximum of the curve, i.e. the Tmax) to more sophisticated parameters (such as production efficiency (OE) / pelvic excretion efficiency (PEE) or normalized residual activity (NORA).

Sufficient information is provided by the renogram and Tmax to distinguish between normal transit (Tmax of about 3 minutes) or transit with a considerable delay (Tmax of 20 minutes).

\subsection{Indications for the examination}

The indications for this type of investigation concern:

1. All uropathies that at diagnosis require an evaluation of the single renal function both during the different surgical phases or conservative treatment and the evaluation of the drainage function.

2. When there is dilation of the collection system, the standard renogram should be supplemented with a diuretic renogram.

3. Previous indirect radionuclide cystography (CRI).

4. Assessment of sustained systemic hypertension. If reno-vascular disease is suspected, Captopril can be used.

5. Kidney trauma.

6. Renal transplant follow-up. Here the tracer dose is higher and rapid acquisition is required.

While there are no contraindications, there are limitations as regards poor kidney function. In this case, accurate estimation of DFR and / or drainage may not be possible.

\subsection{Radio drug}

There are two categories of radiopharmaceuticals:

1. radiopharmaceuticals filtered exclusively by the glomeruli, usable for the calculation of the glomerular filtration rate (GFG);

2. radiopharmaceuticals excreted mainly by the tubules, which can be used for the calculation of the effective renal plasma flow (FPRE);

There are three different tracers that rely on tubular secretion:

- $\quad$ I123 Hippuran (inulin)

- Tc99m Mercaptoacetyltriglycine (MAG3)

- $\quad$ Tc99m Ethylenedicysteine (CE)

and a filtration-dependent tracer:

- Tc99mDiethylene acid-triamino-pentacetic (DTPA)

Tracers reflecting tubular secretion have higher excretion than Tc99m-DTPA following lower activity and higher background ratio.

For these reasons it is preferred to use the Tc99m-DTPA for the estimation of the differential renal function (DRF) especially in newborns. The Tc99mDTPA is useful after a kidney transplant when both blood flow and glomerular filtration rate (GRF) estimation with blood sample analysis is required. 
The kidney of children is immature and renal clearance increases progressively up to about 2 years. Therefore the amount of tracer is particularly low in infants, with a high background activity.

In young children, tubular extraction radiopharmaceuticals (99mTc-MAG3 and 123I-OIH) are preferable to 99mTc-DTPA, because they provide images with less background activity and a better kidney-fundus ratio, especially in infants. It is therefore easier to calculate the separated renal function, even in the presence of still immature kidneys and / or very marked reductions in the function of the single kidney

With the Tc99m-DTPA, the DRF estimate can be inaccurate in early childhood.

\subsubsection{Radionuclide}

Technetium-99m (Tc99m)

Iodine-123 (I123) for Hippuran only

\subsubsection{Drug}

MAG3 (Mercaptoacetyltriglycine)

EC (Ethylenedicysteine)

DTPA (Diethylene acid-triamine-pentacetic)

Hippuran

\subsubsection{Dosage}

Minimum dose:

- $\quad \mathrm{Tc}^{99 \mathrm{~m}}-\mathrm{MAG} 3=15 \mathrm{MBq}$

- $\quad \mathrm{Tc}^{99 \mathrm{~m}}-\mathrm{DTPA}=20 \mathrm{MBq}$

- $\mathrm{THE}^{123}-$ Hippuran $=10 \mathrm{MBq}$

Maximum dose:

- $\quad \mathrm{Tc}^{99 \mathrm{~m}}-\mathrm{MAG} 3=70 \mathrm{MBq}$

- $\quad$ Tc99m-DTPA = 200MBq

- I123-Hippuran $=20-75 \mathrm{MBq}$

\subsubsection{Burden of radiation}

For a 5-year-old child, using the Tc99m-DTPA the effective dose (ED) is 0.54 to $0.82 \mathrm{mSv}$, the lowest figure based on a one-hour urination interval.

For the Tc99m-MAG3 the corresponding figures are respectively from 0.20 to $0.38 \mathrm{mSv}$.

The 5-year ED using I123-Hippuran is $0.41 \mathrm{mSv}$.

\subsection{Execution of the examination}

Position the patient supine, then start the computer and inject the bolus of the radiopharmaceutical.

8.4.1 Image acquisition

Acquisition time: The radiopharmaceutical is injected iv a

"bolus" in the patient already suitably

positioned:

At the same time as the administration the examination begins (duration about 40 minutes. It is a dynamic

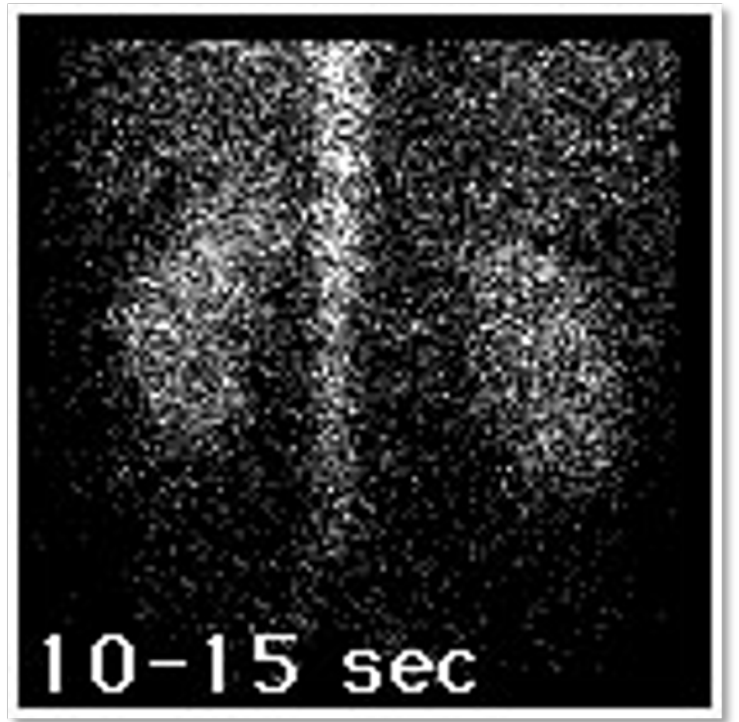

Fig. 7 - Perfusion scintigraphy image.

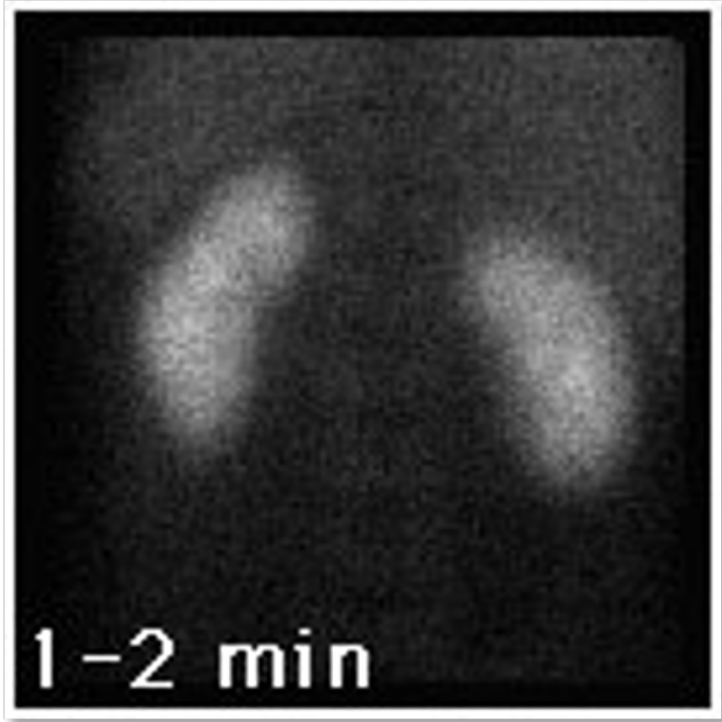

Fig. 8 - Cortical scintigraphic image.

acquisition, consisting of three phases:

1. Perfusion (60 1-second images, for a total of 1 minute).

After the intravenous injection, the abdominal aorta, spleen and kidneys are visualized.

The radioactivity must appear simultaneously in the two kidneys and the intensity must be symmetrical and equal to or greater than that of the spleen.

2. Cortical phase (12 images of 5 seconds, for a total of 1 minute).

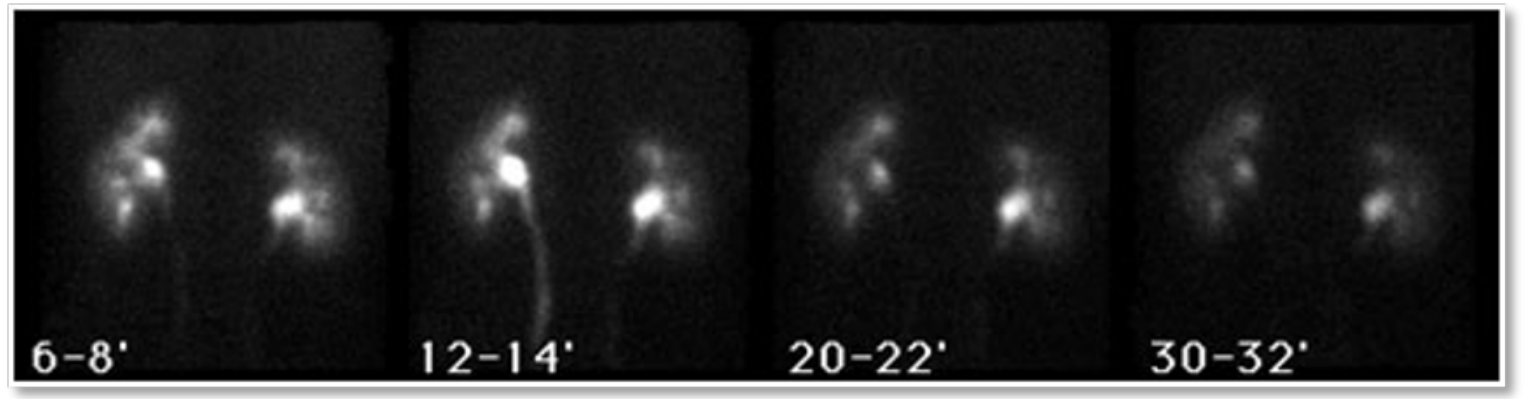

Fig. 9 - Sequential renal scintigraphy. 
Renal uptake must be homogeneous. In this phase the tracer accumulates in the parenchyma; this buildup must be fast and peak within 2-5 minutes.

3. Excretion phase (54 images of 20 seconds, for a total of 18 minutes).

The parenchymal radioactivity progressively decreases and we witness the passage of the radiopharmaceutical into the calyxes, pelvis, ureters and then into the bladder.

Upon completion of the sequential renal scintigraphy, a 3-5 minute static image is usually acquired including the kidneys and bladder to highlight any radioactive urine stagnation in the renal pelvis. It can also be performed in standing position to visualize any renal ptosis.

\subsubsection{Collimator}

Low-energy, high-resolution collimator

\subsubsection{Detector location}

The gamma camera is positioned with the detector facing up

\subsubsection{Activity / time curves (radiophrograms)}

- They graphically represent the radioactivity trend that occurred, during the chosen time interval, within the structures that the operator has identified by tracing the appropriate areas of interest (ROI).

- The standard processing of a sequential renal scintigraphy includes the generation of activity / time curves related to the kidneys, commonly called "renographic curves". They show:

- an initial vascular peak due to the arterial passage of the bolus. This peak, rapidly rising and therefore narrow and sharp, is called "first pass";

- a second peak, wider and slower, due to the extraction of circulating radioactivity by the kidney (expression of glomerular or tubular function depending on the radiopharmaceutical used);

- a descent that mirrors the outflow of radioactive urine from the kidneys.

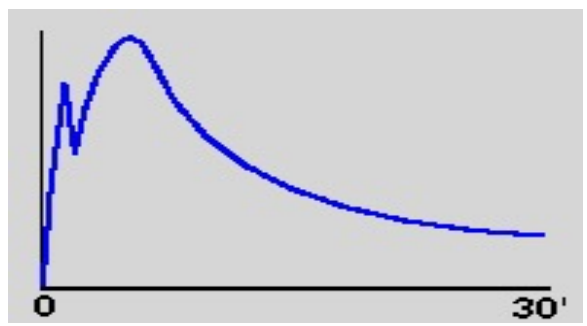

Fig. 10 - Rengoramma

- $\quad$ Since two-dimensional images are acquired (generally in posterior projection), the renal ROIs also "see" the radioactivity distributed in the tissues behind and in front of the kidneys. This overestimation of the counts is corrected by assuming that the radioactivity above and below the kidneys is similar to that of the region immediately adjacent to them. For this, "background" ROIs are used, plotted around the kidneys, which provide data on the course of radioactivity in the adjacent soft tissues. The trend of radioactivity in the "background" area is of a vascular type: after an initial rise, due to the appearance of the radiopharmaceutical, the curve shows a progressive decline resulting from the gradual disappearance of circulating and interstitial radioactivity.

\subsubsection{Quantitative analysis of perfusion}

Kirchner in 1974 introduced the quantitative estimation of renal perfusion, based on the kidney / aorta ratio derived from the curves. Subsequently, different methods of renal flow analysis were developed by means of activity / time curves, based on various parameters, such as the speed of onset of activity in the kidneys or the speed of the activity integral under the curves or time of intravascular renal transit.

- These methods, used above all for the evaluation of acute rejection in transplantation and in the study of renovascular hypertension, have shown evident limits and today have a very marginal role.

It therefore allows you to have:

- Calculation of relative renal function

- Calculation of glomerular filtration rate or effective plasma flow, separately for each kidney

- Study of renal function in patients suffering from diseases that can cause damage to the renal parenchyma, or undergoing nephrotoxic therapies

- Differential diagnosis of renovascular hypertension

- Follow up of patients undergoing renal revascularization

- Obstructive Uropathy:

- Differential diagnosis between simple dilatation and obstruction of the renal excretory passages

- Follow-up of patients on conservative therapy or after corrective intervention

- Morpho-functional study of renal malformations

- Evaluation of possible renal infarction

- Evaluation of patients with renal trauma

- Kidney transplant:

- Functional study and follow-up of the transplant recipient,

- Study of bilateral renal function in the living donor

\section{SEQUENTIAL KIIDNEY} SCINTIGRAPHY WITH DIURETIC TEST (FUROSEMIDE)

The most important indications for this investigation are: Hydronephrosis, Megaureter, Complex malformations (pyelo-ureteral duplicity, renal ectopy, etc.), Renovascular hypertension, Renal transplantation and in general when a separate evaluation of renal function is required. In some cases, urine stagnation occurs in one or both renal pelvises: the failure to flow urine from the pelvis into the ureter may depend on organic or functional factors. Through renal scintigraphy with the furosemide test it is possible to distinguish the organic stenosis from the functional one and pursue the most appropriate therapeutic choice.

A good hydration is essential. For this purpose it is useful to encourage children who are able to cooperate to drink before the examination, for example in the time interval during which the effect of the anesthetic cream is expected, if used. Alternatively, intravenous hydration with pediatric polysaline $(10-15 \mathrm{ml} / \mathrm{kg}$ in the 30 
'preceding the start of the investigation) can be carried out, which is maintained at a slow drop until the end of the procedure. In infants it is advisable to schedule breastfeeding to obtain maximum peace of mind at the time of acquisition.

It should be ensured that the child does not have an allergy to sulfonamides, which can cross-react with furosemide.

\subsection{Radio drug}

The Tc99m-DTPA or the Tc99m-MAG3 is currently used.

\subsubsection{Dose}

$1 \mathrm{mg} / \mathrm{kg}$ with a maximum dose of $20 \mathrm{mg}$.

\subsubsection{Timing of administration}

At the end of the basic sequential renal scintigraphy, having ascertained the pyelic stagnation of the radioactive urine, the diuretic is administered iv, immediately starting the dynamic acquisition of the images (60 images of 20 seconds, for a total of 20 minutes). Images acquired too long after the administration of the diuretic (for example after 1 hour) are not very informative, because even in the case of obstruction there is still a decrease in activity. Another possibility is to evaluate emptying after urination and standing, before injecting furosemide. In this case it is necessary to weigh the risk of not being able to keep the patient immobile in the subsequent phases of the investigation.

\subsection{Patient position}

In the supine position, after the child has been upright for at least five minutes and has emptied his bladder, data should be acquired for at least one minute.

\subsection{Collimator}

For low energies with high resolution parallel holes

\subsection{Processing}

The choice of the first image must coincide with that of maximum vascular activity. The renal areas of interest must be drawn on a sum image, taking care not to exclude portions of the parenchyma or pelvis. Renographic curves must be corrected for the fundus and for fundus subtraction it is advisable to use an area for each kidney, which surrounds it completely or partially. In all cases, care must be taken to avoid undue overlap, especially with a very dilated pelvis or ureter. Background ROI counts should be normalized by area before being subtracted from kidney counts

\subsection{Interpretation}

\section{Qualitative Analysis}

The interpretation, especially as regards the presence or absence of obstruction, must be based on the images of the dynamic phase and on the post-voiding image, as well as on the renographic curves and on any numerical parameters. In the case of comparison with a previous study it is essential that the acquisition and processing are performed in a standardized and repeatable manner. Semiquantitative Analysis

As there is no formal definition of obstruction in the literature, there is no scintigraphic parameter that identifies with certainty the renal units to be subjected to surgery. The parameter on which there is some agreement is the reduction of separate functionality as an in- direct index of significant obstruction. The most common clinical approach involves combined ultrasound and scintigraphic controls, based on which it is decided whether to indicate the surgical correction.

\section{SEQUENTIAL KIDNEY SCINTIGRAPHY WITH CAPTOPRIL TEST}

It is a sequential renal scintigraphy with pharmacological test: it is a method that is used to identify patients with arterial hypertension in whom an intervention to correct the artery stenosis is able to cure hypertension and / or improve function renal.

The examination consists of a sequential renal scintigraphy that is done one hour after the patient has been given an ACE inhibitor (Captopril).

The application of the test is based on the possibility of indirectly highlighting a state of activation of the renin-angiotensin system typical of renovascular hypertension.

This is achieved by pharmacological blockade of the renin-angiotensin compensation system which ensures the constancy of blood pressure in the capillaries of the glomerulus.

In case of stenosis, blocking the compensation, the glomerular filtration is reduced with a consequent worsening of the radionefrogramma compared to the reference one obtained in basal conditions.

\section{PEDIATRIC SCINTIGRAPHY FOR THE EVALUATION OF GLOMERULAR FILTRATE}

Renal clearance of a substance can occur in two processes: glomerular filtration or tubular secretion. Among these processes, the glomerular filtration rate (GFR) is probably the most representative parameter of renal function (6).

For scintigraphic evaluation in children, two methods can be used:

- The "intercepted slope" method, which is based on the determination of the last exponential only by at least two blood samples approximately 2 and 4 hours after the intravenous injection of the radiotracer. Neglecting the first exponentials introduces a systematic overestimation of clearance

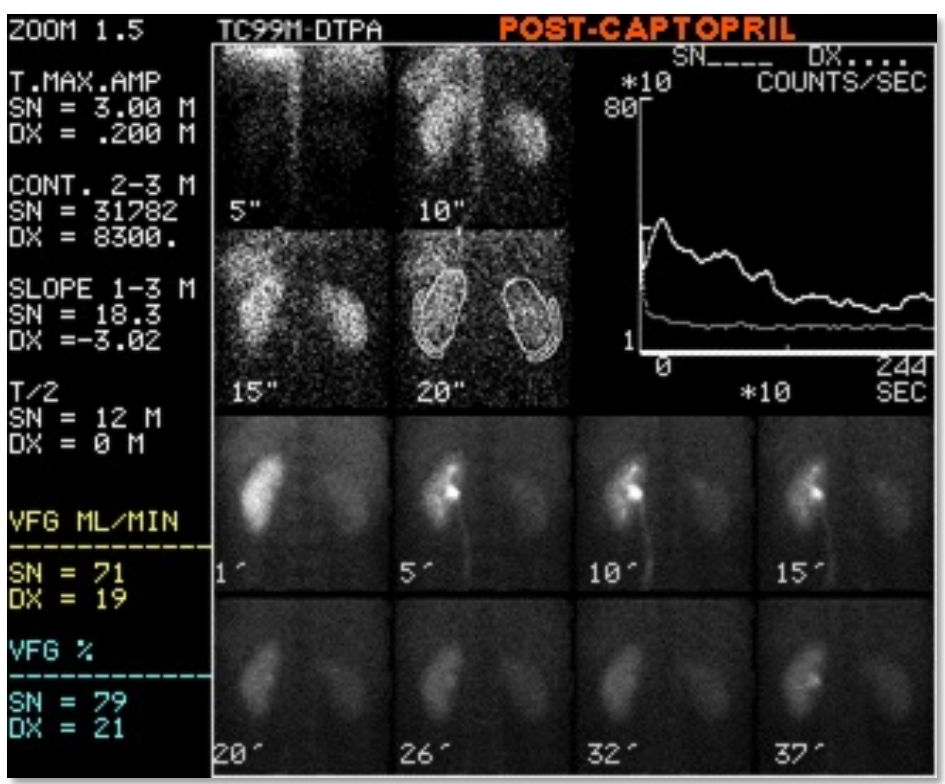

Fig. 11 - Renal scintigraphy with diuretic test 
that can be corrected based on various published algorithms.

- The "volume of distribution" method is based on a single blood sample taken at 2 hours. The main advantage is the need for a single blood sample. This method is valid for children of all ages and the results are identical to those of the method described above, but this approach is not valid in patients with poor renal function (GFR $<30 \mathrm{ml} / \mathrm{min}$ / $1.73 \mathrm{~m} 2)$

\subsection{Instructions for the examination}

\section{Indications}

1. Evaluation and follow-up of chronic renal function in glomerulopathies.

2. Evaluation and follow-up of renal side effects of chemotherapy or nephrotoxic drugs.

3. Estimation of single renal GFR, in conditions such as unilateral or bilateral hydronephrosis, urinary tract infection, with or without associated vesicourethral reflux, small kidney, single kidney, double kidney, urethral valves, pre and post follow-up.

4. Determination of GFR can be considered whenever there is suspicion of renal failure, even while plasma creatinine is in the normal range.

Contraindications

There are no contraindications.

\subsection{Radio drug}

\subsubsection{Radionuclide}

Chromium 51 (Cr51)

Technetium 99m (Tc99m)

\subsubsection{Drug}

Ethylenediaminateracetic acid (EDTA)

Diethylenetriaminopentaacetic acid (DTPA)

Cr51-EDTA is probably the best tracer for the evaluation of GFR, in relation to the close link of Chromium to EDTA. The Tc99m-DTPA is a valid alternative.

11.2.3 Dosage

- Cr51-EDTA

Minimum dose $=0.074 \mathrm{MBq} / \mathrm{Kg}$

Maximum dose $=3.7 \mathrm{MBq}$

- Tc99m-DTPA

The dose administered is scaled on a base body surface.

Maximum dose $=37 \mathrm{MBq}$.

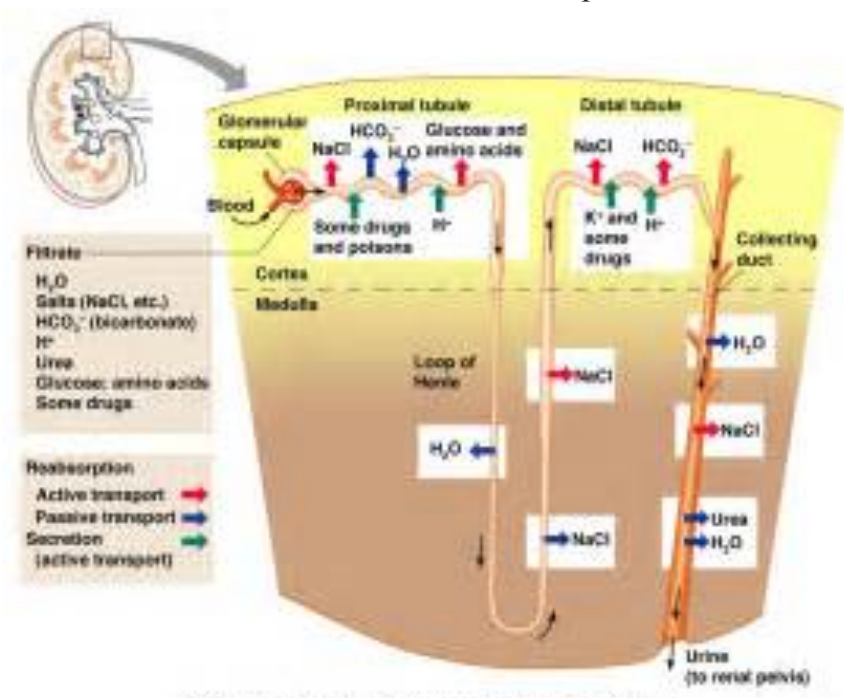

Fig. 12 - Renal function diagram
12 PEDIATRIC SCINTIGRAPHY

FOR RENAL PARENCHYMAI

ANOMALIES WITH TC99M-DMSA

(DIMERCAPTOSUCCINIC ACID)

It is a non-invasive method which, through the administration of radiopharmaceuticals that fix themselves in the cortical of the kidneys in a sufficiently stable way to allow to visualize and "quantify" the functioning renal parenchyma. Particularly suitable for the study of the estimate of relative renal function, in cases of acute pyelonephritis (more sensitive than intravenous urography and ultrasound), for diagnosis, localization and follow-up (identification of parenchymal damage, progression of damage in case of of relapsing urinary infections.

Identification of ectopic kidney or confirmation of renal agenesis); for renal morphological study in case of malformation; for the differentiation of renal masses (fetal lobulation, dromedary hump kidney, compensatory lobar hypertrophy, hypertrophic Bertin's spine) versus secondary space-occupying lesions. It also allows the evaluation of kidney trauma. The lesions are non-specific, as similar lesions can be found in renal abscesses, cysts, double kidney and hydronephrosis: the combination of ultrasound and scintigraphy with DMSA allows a better differentiation between these clinical situations. In case of marked hydronephrosis, the tracer can accumulate in the renal cavity causing difficulties in interpreting cortical images.

\subsection{Indications in the examination} Indications

A. Detection of renal parenchyma abnormalities

B. Detection of kidney sequelae, six months after acute infection;

C. Detection of acute pyelonephritis;

D. Detection of associated anomalies (abnormal double kidney, small kidney, ..);

E. Detection of ectopic kidney;

F. Confirmation of non-functioning polycystic kidney.

\subsection{Radiopharmaceutical \\ 12.2.1 Radionuclide \\ Technetium 99m (Tc99m)}

\subsubsection{Medication}

DMSA (dimercaptosuccinic acid)

Although several detectors are available for cortical imaging, the most appropriate tracer for this is Tc99mDMSA. This tracer is picked up by the tubular cells of the pars recta (see fig. 11), directly by the peritubular vessels.

Dynamic detectors with a high excretion rate, such as the Tc99m-MAG3 or Tc99m-CE, give less precise information on regional cortical anomalies and constitute only a second choice of the tracer.

\subsubsection{Dosage}

Minimum dose: $15 \mathrm{MBq}$

Maximum recommended dose: $100 \mathrm{MBq}$

\subsection{Image acquisition}

\subsubsection{Acquisition time}

Images should be acquired 2 to 3 hours after tracer injection. 
Late images (4 to 24 hours) are useful if significant hydronephrosis is present.

\subsubsection{Collimator}

A high or very high resolution collimator is required. The pin-hole collimator (2-3 mm opening) can be useful, especially in newborns.

\subsubsection{Position of the detector}

The camera must be positioned with the collimator upwards.

\subsubsection{Patient position}

The patient is supine to minimize the difference in renal depth and help minimize movement.

\subsubsection{Projections}

Posterior and posterior oblique views are recommended; an additional anterior view can be performed in the case of an ectopic kidney in the pelvis or a horseshoe kidney.

\section{PEDIATRIC SCINTIGRAPHY FOR THE RESEARCH OF ECTOPIC GASTRIC MUCOSA IN MECKEL'S DIVERITICLE}

Ilieal or Meckel's diverticulum is a congenital anomaly due to the presence of a residue of the omphalomesenteric duct, which is organized as a diverticulum, a full-bottom blind bag ilealFirst described by Fabricius Hildanus in 1598, owes its name to the German anatomist Johann Friedrich Meckel, which demonstrated its characteristics embryological is pathological in 1809 . Meckel's diverticulum occurs in about 1-2\% of people. Clinical presentation can be characterized more often by bleeding $(25-50 \%)$ acute or chronic relapsing, due to ulceration of the ileal mucosa adjacent to the heterotopic gastric mucosa; bleeding can occur in the form ofmelenaas heterotopic gastric tissue can digest blood. A patient with Meckel's diverticulum has a 4-6\% higher risk of developing abdominal complications, including: obstruction, intussusception, inflammation (meckelitis) and perforation.

Rarely symptomatic after 10 years, more often thehemorrhageaffects children under the age of 2, while obstruction is more common in adults. Complications are more frequent in males, with ratios ranging from 1.8: 1 to $3: 1$.

The obstruction can be caused by volvulus, intussusception, herniation (Littre's hernia), arterial compass, chronic diverticulitis, lithiasis, fibrous bands, tumors. Meckelitis accounts for $20 \%$ of symptomatic cases, with aclinical picture which can often be confused with a appendicitisacute. The perforation takes place fordiverticulitisulceration secondary to gastric mucosa, foreign bodies, bezoars, trauma, tumor.

Tumors in Meckel's diverticulum are rare, presenting with incidenceof $0.5-1.9 \%$. They can be benign (lipoma, hamartoma) or malicious: carcinoids (44\%), mesenchymal tumors $(35 \%)$ like the GIST $(12 \%)$ and the leiomyosarcoma, adenocarcinomas $(16 \%)$ e small round cell desmoplastic tumor.

Treatment is invariably surgical, with resection of the pathological tract.

It is a method that exploits the ability of the gastric mucosa to intensely concentrate $99 \mathrm{mTc}$-pertechnetate. The presence of abdominal concentration of this radiopharmaceutical extra-gastric indicates the probable

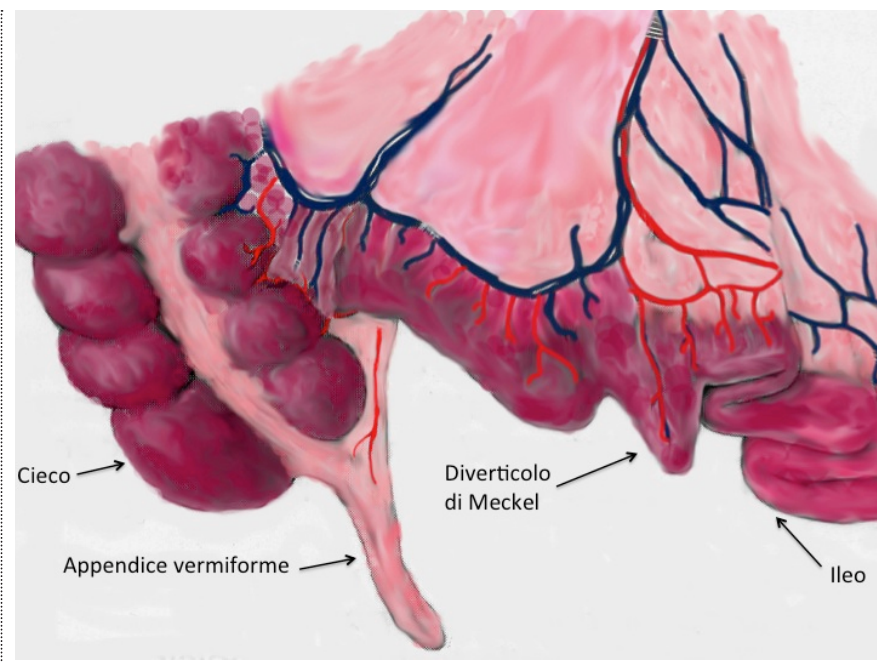

Fig. 13 - diverticulum of meckel

presence of ectopic gastric mucosa in a Meckel's diverticulum.

\subsection{Preparation}

The patient must have been fasting for at least 12 hours and should not have had a colonoscopy and / or barium enema in the previous 48 hours.

To increase the sensitivity of the investigation, a pretreatment with a drug capable of blocking the $\mathrm{H} 2$ receptors of histamine is performed. In this way the uptake of the radiopharmaceutical in the gastric mucosa is favored and its secretion is blocked. 13.2 Dosage $20 \mathrm{mg} / \mathrm{kg} /$ day for 2 days with a total dose of 120 $\mathrm{MBq}(\approx 3.2 \mathrm{mCi}) .0$. In case of urgency, the scintigraphic investigation can be performed one hour after the administration of $1 \mathrm{mg} / \mathrm{kg}$ of iv ranitidine, up to a maximum of $50 \mathrm{mg}$, infused over 20-30 minutes.

\subsection{Execution}

The examination involves the intravenous injection of $300-450$ of $99 \mathrm{mTc}$-pertechnetate to the supine patient, with the head of the gamma camera positioned against the abdomen, and the acquisition of 1 minute images for 1 hour. At the end of the dynamic phase, if hypercaptant areas of unsafe interpretation are highlighted, static images are acquired in different projections, possibly after administering an iv diuretic (furosemide) to eliminate radioactivity from the renal excretory tracts. It is a non-invasive method that exposes to a low dose of ionizing radiation (effective dose $=2-3 \mathrm{mSv}$ ).

The survey is able to quickly and non-invasively highlight the presence of gastric mucosa in an abnormal location.

In the normal subject there is a clear concentration of the radiopharmaceutical in the stomach and in the renal excretory tract. An overcaptive area that appears simultaneously with the stomach and that does not move significantly with peristalsis is attributable to ectopic gastric mucosa

The following image shows the scintigraphic picture of a patient with a Meckel's diverticulum in the lower right abdominal area The hypercaptive area is easily recognizable which has been surrounded with a red area of interest (1) appearing at the same time as the stomach, surrounded by the blue area of interest (2). The relative activity-time curves confirm that the modalities of concentration of the radiopharmaceutical within these 2 areas can be superimposed. 


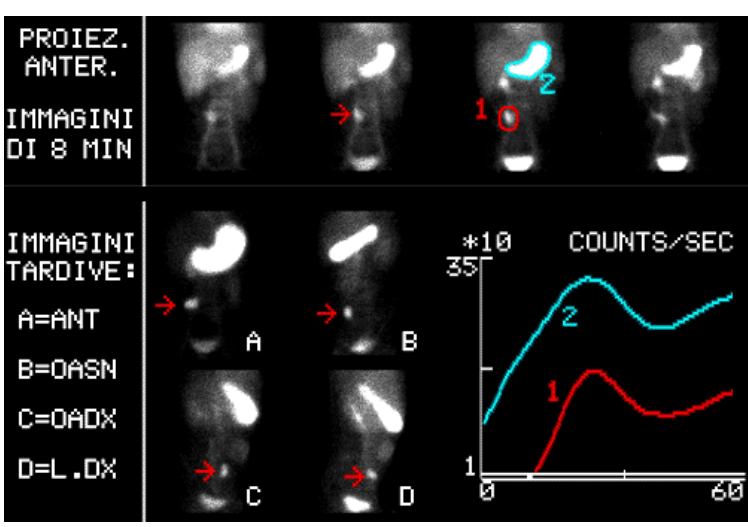

Fig. 14 - Meckel's diverticulum scan

The following figure shows the appearance in the abdomen of a small area of uptake on the transverse umbilical, in the right paramedian, after visualization of the stomach. This area progressively concentrates the pertechnetate.

The corresponding activity / time curve shows a trend similar to the curve relating to the gastric region.

In this clinical case a scintigraphy with administration of diuretic $(0.75 \mathrm{mg} / \mathrm{kg}$ intravenous furosemide) is also subsequently performed in order to exclude its relevance to renal excretory tracts; in fact, the picture remains substantially unchanged to such an extent that the diverticulum, in lateral projection, is located in the middle planes of the abdomen.

Scintigraphy with Tc-99m-pertechnetate which demonstrates an uptake area in the mesogastric region suggestive of Meckel's diverticulum with ectopic gastric mucosa (fig. 16)

\section{VENTILATORY AND}

PERFUSIONAL PEDIATRIC

\section{PULMONARY SCINTIGRAPHY}

Congenital pathologies and malformations that can lead to impaired pulmonary perfusion can be cardiac, large venous and arterial vessels, pomonar circulation vessels, as well as secondary to more complex malformations affecting multiple organs and systems. Congenital malformations are, in fact, very heterogeneous both for the type of organ affected and for the severity and etiology; if considered individually, they represent quite rare events, the frequency of which varies from one case out of 300 born for heart disease, one case out of 2500-3500live births for congenital diaphragmatic hernia. pulmonary perfusion scintigraphy is a nuclear imaging technique which, in clinical practice, finds application in all pathologies that cause alteration of perfusion, but its main indication is pulmonary embolism. this is a condition of clinical urgency which needs to be recognized quickly and which in PPS appears with the characteristic picture of reduced perfusion with mono or plurisegrmentary extension, lobar or an entire lung. The most accurate morphological imaging of the circulation of the great vessels, of the heart and of the pulmonary circulation is obtained with CT angiography, a morphological technique that, through the use of the contyrasto medium, allows to evaluate course, caliber, altered vascular communications and defects of the heart cavities. Nuclear medicine, in the evaluation of the pulmonary perfusion of the pediatric patient with cardiac and non-congenital anomaly, has an emerging role both in the immediate post-operative and in the follow-up of these patients, with the advantage of a low exposure to ionizing radiation and therefore the possibility to retake the exams in a short time. In fact, SPP does not cause side effects, is well tolerated and allows to avoid sedation of the pediatric patient. It is possible to perform planar and tomographic acquisitions for a better evaluation of the case, without further administration of radiopharmaceutical and therefore without increasing exposure to ionizing radiation, in pediatric age, being able to make a diagnosis without excessively irradiating the patient is an essential requirement. in the evaluation of the pulmonary perfusion of the pediatric patient with cardiac and non-congenital anomaly, it has an emerging role both in the immediate postoperative and in the follow-up of these patients, with the advantage of a low exposure to ionizing radiation and therefore the possibility of recovering exams in a short time. In fact, SPP does not cause side effects, is well tolerated and allows to avoid sedation of the pediatric patient. It is possible to perform planar and tomographic acquisitions for a better evaluation of the case, without further administration of radiopharmaceutical and therefore without increasing exposure to ionizing radiation, in pediatric age, being able to make a diagnosis without excessively irradiating the patient is an essential requirement. in the evaluation of the pulmonary perfusion of the pediatric patient with cardiac and non-

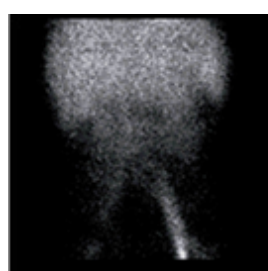

$1-3^{\prime}$
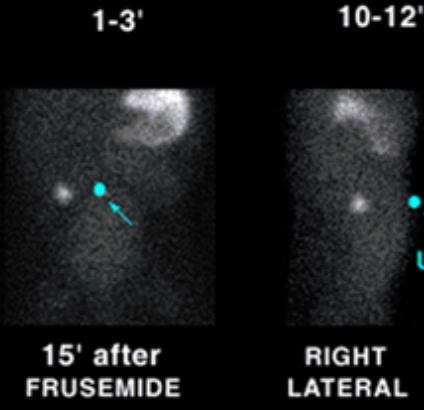

UMBILICUS
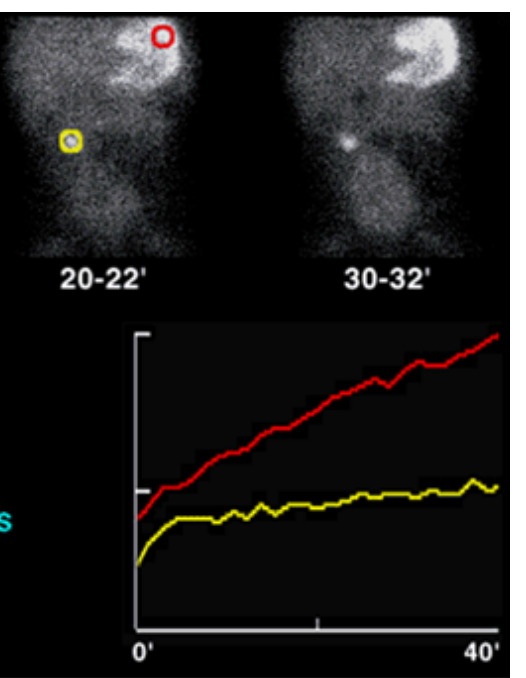

Fig. 15

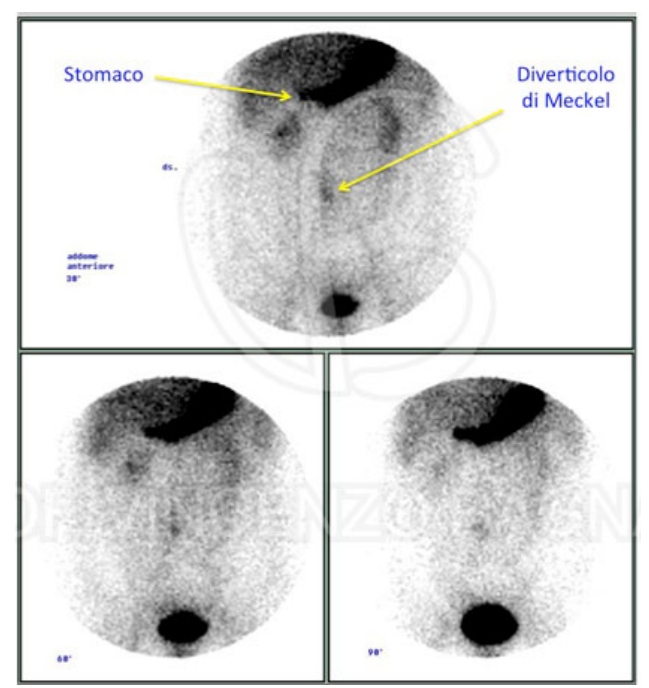

Fig. 16 
congenital anomaly, it has an emerging role both in the immediate post-operative and in the follow-up of these patients, with the advantage of a low exposure to ionizing radiation and therefore the possibility of recovering exams in a short time. In fact, SPP does not cause side effects, is well tolerated and allows to avoid sedation of the pediatric patient. It is possible to perform planar and tomographic acquisitions for a better evaluation of the case, without further administration of radiopharmaceutical and therefore without increasing exposure to ionizing radiation, in pediatric age, being able to make a diagnosis without excessively irradiating the patient is an essential requirement. it has an emerging role both in the immediate post-operative period and in the follow-up of these patients, with the advantage of a low exposure to ionizing radiation and therefore the possibility of retaking the exams in a short time. In fact, SPP does not cause side effects, is well tolerated and allows to avoid sedation of the pediatric patient. It is possible to perform planar and tomographic acquisitions for a better evaluation of the case, without further administration of radiopharmaceutical and therefore without increasing exposure to ionizing radiation, in pediatric age, being able to make a diagnosis without excessively irradiating the patient is an essential requirement. it has an emerging role both in the immediate post-operative period and in the follow-up of these patients, with the advantage of a low exposure to ionizing radiation and therefore the possibility of retaking the exams in a short time. In fact, SPP does not cause side effects, is well tolerated and allows to avoid sedation of the pediatric patient. It is possible to perform planar and tomographic acquisitions for a better evaluation of the case, without further administration of radiopharmaceutical and therefore without increasing exposure to ionizing radiation, in pediatric age, being able to make a diagnosis without excessively irradiating the patient is an essential requirement. with the advantage of a low exposure to ionizing radiation and therefore the possibility of retaking the exams in a short time. In fact, SPP does not cause side effects, is well tolerated and allows to avoid sedation of the pediatric patient. It is possible to perform planar and tomographic acquisitions for a better evaluation of the case, without further administration of radiopharmaceutical and therefore without increasing exposure to ionizing radiation, in pediatric age, being able to make a diagnosis without excessively irradiating the patient is an essential requirement. with the advantage of a low exposure to ionizing radiation and therefore the possibility of retaking the exams in a short time. In fact, SPP does not cause side effects, is well tolerated and allows to avoid sedation of the pediatric patient. It is possible to perform planar and tomographic acquisitions for a better evaluation of the case, without further administration of radiopharmaceutical and therefore without increasing exposure to ionizing radiation, in pediatric age, being able to make a diagnosis without excessively irradiating the patient is an essential requirement.

\subsection{Instructions for the examination}

- Primary pulmonary or pulmonary vascularity anomalies

- Congenital anomalies of the heart and great vessels (pre and / or post surgery)

- Infectious or post-infectious lung lesions (e.g. bronchiectasis)

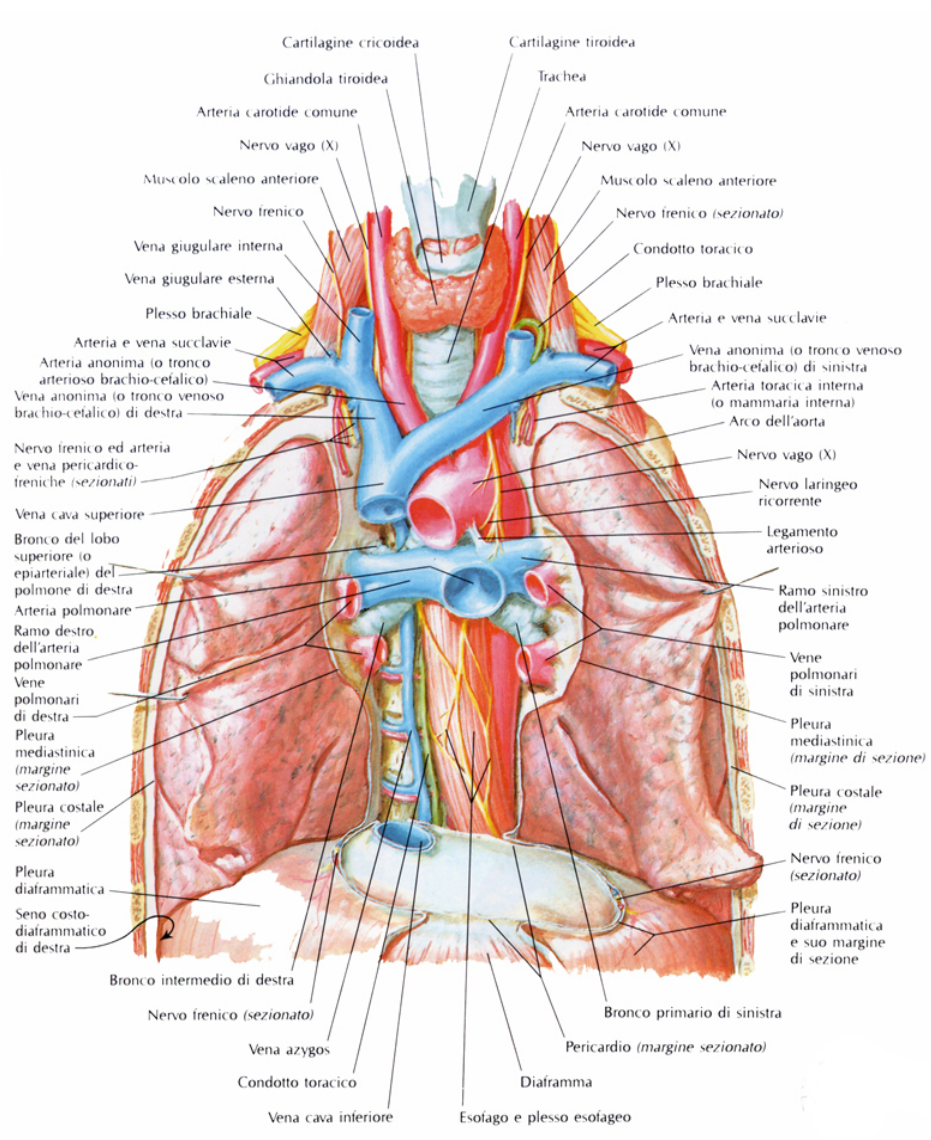

Fig. 17 - Anatomical structure of the lungs

- Pre- and post-surgical evaluation of regional function

- Cystic fibrosis

- Inhalation of foreign bodies

- Diagnosis and quantification of a right-to-left shunt.

- Rare or experimental indications:

- Suspicion of pulmonary embolism

- Suspicion of abnormalities in the mucociliary function

- Pulmonary toxicity assessment

- Assessment of aerosol distribution before administering nebulised drugs

In the case of primary respiratory diseases, such as the presence of a foreign body or cystic fibrosis, there is an almost perfect match between ventilation and perfusion due to the very efficient mechanism of regional vasoconstriction.

However, in primary vascular diseases (for example, embolism or pulmonary abnormalities) perfusion scintigraphy is preferred. Therefore the perfusion technique must be the first step in all common indications. When perfusion scintigraphy is normal, a ventilation scan is not necessary, especially in departments where ventilation should be performed with Tc99m-aerosol or Tecnegas, as it is a time-consuming examination.

When perfusion is abnormal, it is up to the physician to determine whether or not a lung abnormality is likely and whether a ventilatory scan is required.

Most indications for pediatric lung scans are primary ventilatory abnormalities, for which ventilation would simply duplicate the information provided by perfusion. 


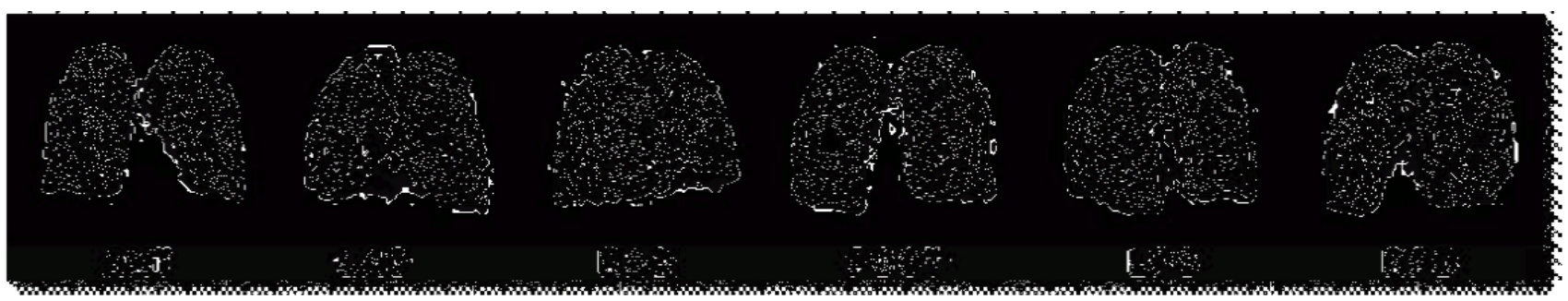

Fig. 18 - Lung perfusion scan

\subsection{Perfusional scintigraphy}

Lung perfusion scintigraphy provides a visual representation of the regional distribution of pulmonary blood flow at the time of injection of the radiopharmaceutical. The investigation is devoid of significant side effects and is well tolerated even in patients suffering from severe lung or heart disease. The procedure involves a simple intravenous injection; the number of injected particles, which varies according to weight, is very small compared to the number of pulmonary capillaries; microembolization affects less than $1 \%$ of pulmonary capillaries, is transient and resolves within a few hours (T1 / 2eff. $=5.3$ hours). Macro-aggregates or microspheres of albumin labeled with $99 \mathrm{mTc}$ are administered intravenously, which cause, after passing through the right heart,

It is a method that allows to evaluate the perfusion of the lung by the pulmonary arteries. The high degree of safety, the relative simplicity of execution and the low cost, combined with a high predictivity make it the first choice imaging investigation in the suspicion of pulmonary embolism.

Microembolization occurs at the first passage through the pulmonary bed and, in the absence of significant right-to-left shunt, the particles do not reach the systemic circulation.

The presence of right-to-left shunt is not an absolute contraindication, so much so that the investigation, after an appropriate reduction in the dose of particles, is also performed for the study of pulmonary perfusion in patients suffering from complex congenital heart disease, even in pediatric age, o to evaluate the extent of intrapulmonary shunt in patients with liver disease

\subsubsection{Radio drug}

For this type of investigation human albumin macro aggregates (MAA) are used. These are preparations for heat denaturation of human albumin and conjugate with $99 \mathrm{mTc}$.

Dimensions: 90\% between 5-90 $\mu \mathrm{m}$, most between 10 $40 \mu \mathrm{m}$.

The biological half-life ranges from 2 to 9 hours depending on the size; after being degraded and reduced in size, they pass the pulmonary filter and reach the systemic circulation from where they are removed by means of the reticulo-endothelial system of the spleen and liver.

The number of particles must be reduced compared to the adult, because the child has a smaller pulmonary vascular bed. For guidance, reference can be made to the following table:

Weight Number of macro-aggregates

$<10 \mathrm{~kg} 10,000-50,000$

$10-20 \mathrm{~kg} 50,000-150,000$

$20-35 \mathrm{~kg} 150,000-300,000$

$35-50 \mathrm{~kg} 300,000-500,000$
14.2.2 Radionuclide

Technetium 99m (Tc99m)

\subsubsection{Drug}

Human albumin macroaggregates (MAAs) are used.

\subsection{Ventilatory scintigraphy}

The administration by air of inert gases, aerosols or of ultrafine micro particles marked with technetium, allows the study of regional ventilatory function.

The methodology of execution and the information obtainable vary according to the radiopharmaceuticals used.

\subsubsection{Radio drug}

Inert gases are used that allow the evaluation of ventilation in the various lung districts.

\subsubsection{Radionuclide}

Technetium 99m (Tc99m)

\subsubsection{Drug}

DTPA (Diethylene-triamino-pentacetic acid)

Tecnegas (dispersion of particles marked with Carbon)

Kr81m (Kripton $81 \mathrm{~m}$ ): gas added to breathed air

Tc99m-aerosol: Ventilation studies can be performed safely by inhaling radioactive droplets (aerosols) labeled with Tc99m (9). Different aerosols are used, but DTPA is preferred for pediatric scintigraphy because it allows rapid renal clearance, which lowers the radiation burden. Even small droplets $(0.8-15 \mu \mathrm{m})$ have a mass large enough to be affected by turbulent ventilatory flow and can easily distribute within the oral and bronchial mucosa.

Ingestion of saliva can cause a high gastric signal with possibly difficult visualization of the left base of the lung. The tracer administered must be rapid enough to avoid frequent swallowing.

Tc99m-Tecnegas: the name Tecnegas was invented in November 1984 to describe the new agent for fan studies, produced by a special device (Tecnegas generator). Tecnegas consists of flat hexagonal crystals of technetium wrapped in several layers of graphite sheets, which completely isolates the metal from the external environment. Each particle is 5-30 nm wide and $3 \mathrm{~nm}$ thick, and is suspended in an Argon vector as a consequence of its production.

The particles are clearly hydrophobic, which is of considerable importance for a pulmonary agent as they will be repelled by an aqueous surface and water vapor. Almost all activities have been shown to extend beyond the sixteenth division of the bronchial tree. In children, due to lack of cooperation, Tecnegas can also accumulate in the proximal bronchi and trachea. When a significant amount of the tracer is trapped in 
the oropharynx and is swallowed with saliva, this can cause artifacts, such as those seen with radiolabelled aerosols.

Krypton-81m: Kr81m is a gas that allows for easy implementation of constant regional ventilation during a continuous inhalation study. Due to its short half-life of 13 seconds, a significant fraction of the gas is no longer radioactive during the time it takes for the $\mathrm{Kr} 81 \mathrm{~m}$ to mix with the air in the alveoli. The slower the regional ventilation (i.e. the exchange of local air in and out of the alveoli), the smaller the fraction of the still radioactive $\mathrm{Kr} 81 \mathrm{~m}$ that reaches the alveoli. Therefore, the intensity of the signal on the scintigraphic image is linked to the exchange of air entering and leaving the alveoli, also called specific ventilation. The regional distribution of $\mathrm{Kr} 81 \mathrm{~m}$ does not represent pure ventilation, but a mixture of ventilation and volume. This proportionality is not linear, and ventilatory mixing can be very fast, sufficient to minimize radioactive decay. This condition is often present in infants and young children, and can cause difficulties in identifying reduced ventilation regimes. However, these disadvantages are balanced by the Kr81m's unique ability to study the breathing tide with a negligible burden of radiation: furthermore, the acquisition of ventilatory studies using the Kr81m is simple, without requiring special patient cooperation. The Kr81m generator ( $\mathrm{Rb} 81 / \mathrm{Kr} 81 \mathrm{~m})$ produces the Krypton diluted in atmospheric air; the mother isotope Rubidium ( $\mathrm{Rb} 81$ ) is obtained as a product of the cyclotron. The parent remains active enough for clinical purposes for approximately 12 hours after calibration. Unfortunately, it can be quite difficult to buy $\mathrm{Kr} 81 \mathrm{~m}$ generators these days, as most companies no longer manufacture them.

\subsection{Administration of the tracer}

Tc99m-MAA: Tc99m-MAA is administered intravenously. This tracer must be stored in its glass bottle and gently shaken before drawing it via a syringe. It can then be injected without waiting, to avoid adhesion of the Tc99m-albumin particles in the inner wall of the syringe.

When a ventilation and perfusion scan is to be performed in the same session, the two tracers should be administered with the patient in the same position. If radioaerosol-labeled Tc99m or Tecnegas are used for ventilatory scanning, perfusion should be performed first.

If $\mathrm{Kr} 81 \mathrm{~m}$ gas is used for the ventilatory scan, this can be acquired prior to perfusion or both exams can be performed simultaneously as a dual isotope scan.

Care should be taken in calculating the amount of particles administered, considering that newborns have pre-capillary vessels ten times smaller than adults. The number of particles should be kept as low as possible to embolize no more than $0.1 \%$ of the total capillary vessels of the lung. If a severe reduction (more than 50\%) of the vascular bed of the lung is known or suspected, a proportional reduction in the number of particles to be injected is mandatory. A good rule of thumb is to prepare the tracer in order to obtain a known amount of activity (for example, 1-1.5 MBq) for every 5000 particles present in the vial and to inject $1 \mathrm{MBq} / \mathrm{Kg}$ for the body weight.

It is very important that the physician and nuclear medicine technician are aware of congenital heart and great vessel abnormalities, as well as possible different postsurgical anatomy.
Tc99m: the use of Tc99m-aerosol is important to avoid contamination from both breathed air and air escaping from the aerosol. The radiopharmaceutical can be administered in front of the gamma camera via a surgical mask over the nose and mouth of children under 2 years of age or via a nebulizer hood. The administration of the aerosol is stopped after 2 minutes.

The minimum activity within the lungs should be no less than $10 \mathrm{MBq}$, to allow for sufficient statistical account. When ventilation and perfusion are to be performed within the same session, the activity administered for the ventilatory scan should be approximately one third of the Tc99m-MAA dose for perfusion. It is necessary to test the procedure in each nuclear medicine department and to modify the dose inserted in the nebulizer to correct for differences in the efficiency of the different aerosol equipment.

Tc99m-Tecnegas: the patient uses a disposable breathing set and at the end of inspiration it is strongly recommended to hold the breath for 5 seconds because it increases the retention of the tracer for each breath from $20 \%$ to $80 \%$.

If possible, patients should be supine during inhalation. It is best to administer the tracer in a separate, wellventilated room other than the scan chamber, to prevent environmental contamination and an increase in background activity. The administration of Tecnegas into the scanning chamber is not ideal, and can only be done if there is no alternative.

Krypton- $81 \mathrm{~m}$ : Kr81m is a gas eluted with humidified air through the rubidium generator and administered by continuous inhalations during image acquisition, mixed with atmospheric air. In infants and young children, the amount of breathing is low compared to the flow of $\operatorname{Kr} 81 \mathrm{~m}(21 / \mathrm{min})$, so that a constant concentration is obtained simply by placing the tip of the tube near the nostrils; excess gas that is not inhaled can be removed by simply blowing it out of the field of view with a fan.

Due to the rapid decay of $\mathrm{Kr} 81 \mathrm{~m}$, environmental contamination is not a problem and the amount of scattered radiation is very low compared to other radioisotopes for ventilatory studies and with a chest X-ray.

\subsection{Dosimetry}

- Minimum activity: 15 MBq Tc99m-MAA

- The maximum activity varies between 100 and $160 \mathrm{MBq}$, according to different national companies and local regulations.

- The administered dose should be divided on the basis of the body surface area.

\subsection{Acquisition of images}

14.6.1 Perfusion study

The patient lies in the supine position, if possible lying directly over the collimator. Acquisition begins a few minutes after the injection iv. A high resolution parallel hole low energy collimator is used.

Acquisition parameters:

- Static images on a $256 \times 256$ or $128 \times 128$ matrix

- Zoom: adapted to the size of the patient (avoid too small images)

- Total count between 200,000 and 500,000 counts per image

If only the calculation of relative lung perfusion is required, the posterior projection alone is usually sufficient. To quantify the severity of a right-to-left shunt, a posterior projection image including the lungs and the 
skull can be acquired for the calculation of the shunt index. Alternatively, whole body images can be acquired in anterior and posterior projection at a speed of $30 \mathrm{~cm}$ / minute.

\subsubsection{Ventilation and perfusion spark}

The order of acquisition of the two investigations must be decided case by case on the basis of the clinical question and the degree of collaboration of the patient, but it is advisable to perform them on the same day. The waiting time must be a few minutes for the 99mTc-DTPA, while the Technegas is retained much longer after administration and does not impose close constraints

\section{SPARK WITH 123I / 131II-MIIBG}

\subsection{Indications}

Imaging of neuroendocrine tumors and their metastases: in particular pheochromocytoma, functioning and non-functioning paraganglioma, neuroblastoma, ganglioneuroblastoma, ganglioneuroma, carcinoid tumors, medullary thyroid carcinoma, Merkel cell tumors, MEN2 syndromes.

The indications for performing 123I / 131I-MIBG scintigraphy are: Identification and localization of the primary tumor and its metastases (staging), mainly in pheochromocytoma, functioning paraganglioma and neuroblastoma.

Follow-up of patients with known disease (re-staging). Monitoring of the effects of therapies (surgery, radiotherapy, chemotherapy and 131I-MIBG therapy) by measuring the variation in the intensity of uptake and the number of lesions.

Evaluation of tumor uptake preliminary to 131I-MIBG therapy.

Confirmation of suspected neuroendocrine tumors: urinary catecholamines or plasma / urinary metanephrines ( 2 out of 3 conditions required). - Children with expansive adrenal lesion with radiological suggestive aspects of neuroblastoma (calcifications). If the lesion has been diagnosed by ultrasound in the pre-natal period, an observation period of at least 6 months after birth is required before performing the scintigraphy to demonstrate the effective proliferative attitude of the adrenal lesion.

- Children with acute cerebellar encephalopathy (opso-myoclonus-ataxia), for the search for the site of occult neuroblastoma, the cause of the typical neurological syndrome.

\subsection{Contraindications}

Inability of the patient to cooperate

\subsection{Radio drugs and administered activities}

123I- or 131I-MIBG are marketed ready for use in solution for ev use. For dosimetric considerations and for the quality of the images obtained, 123I-MIBG is the radiopharmaceutical of choice, especially in children and women of childbearing age. The dose must refer to Legislative Decree $187 / 2000$. In general, in pediatric patients the activity to be injected must be calculated according to what is indicated by the EANM Pediatric Group:

minimum activity $20 \mathrm{MBq}$ for $123 \mathrm{I}-\mathrm{MIBG}$ and 35 MBq for 131I-MIBG

maximum activity $400 \mathrm{MBq}$ for 123I-MIBG and 80 $\mathrm{MBq}$ for 131I-MIBG.

In patients with pheochromocytoma and functional paraganglioma, the absorption of MIBG into the granules of chromaffin cells could, rarely, cause rapid secretion of catecholamines with consequent hypertensive crisis. In such patients, ECG and blood pressure monitoring may be indicated during administration. The MIBG should be administered intravenously. In the case of small children, the rate of infusion and the total amount of volume administered should be agreed with the pediatrician. Dosimetry Absorbed dose per unit of activity administered (mGy / MBq)

\subsection{Acquisition}

Patients should empty their bladder prior to imaging. The gamma camera, possibly a large field of view for whole-body imaging, should be equipped with a parallel hole high energy collimator for 131I-MIBG and a high resolution low energy collimator for 123I-MIBG. The energy window must be set at $20 \%$ around the photopeaks of 131I (346 KeV) and 123I (159 KeV). Planar and SPECT images must be acquired at 24-48 for the 131I-MIBG with possible repetition at 72 hours in case of interpretative doubts especially at the abdominal level (interference of intestinal activity); between 20 and 24 hours from the injection for 123IMIBG with possible repetition at 48 hours in case of interpretative doubts. In these cases it is important to obtain at least two series of images of the different body districts, preferably with a tomographic acquisition. For the 131IMIBG: whole-body images with anterior and posterior acquisitions and speed of $4 \mathrm{~cm} /$ minute; alternatively anterior and posterior planar images of the head, neck, thorax, abdomen, pelvis, arms and lower limbs: at least

Dosimetria

\begin{tabular}{|l|c|c|c|c|c|c|}
\hline \multicolumn{7}{|c|}{ Dose assorbita per unità di attività somministrata (mGy/MBq) } \\
\hline Organo & \multicolumn{7}{|c|}{${ }^{\text {I31 I-MIBG }}$ I-MIBG } \\
\hline & Adulti & 15 anni & 5 anni & Adulti & 15 anni & 5 anni \\
\hline Surreni & 0.17 & 0.23 & 0.45 & 0.017 & 0.022 & 0.045 \\
\hline Vescica & 0.59 & 0.73 & 1.70 & 0.048 & 0.061 & 0.084 \\
\hline Cuore & 0.072 & 0.091 & 0.20 & 0.018 & 0.024 & 0.055 \\
\hline Reni & 0.12 & 0.14 & 0.30 & 0.014 & 0.017 & 0.036 \\
\hline Fegato & 0.83 & 1.10 & 2.40 & 0.067 & 0.087 & 0.18 \\
\hline Milza & 0.49 & 0.69 & 1.70 & 0.020 & 0.028 & 0.066 \\
\hline Ovaie & 0.066 & 0.088 & 0.23 & 0.0082 & 0.011 & 0.025 \\
\hline Tiroide & 0.050 & 0.065 & 0.18 & 0.0056 & 0.0073 & 0.019 \\
\hline Dose efficac (mSv/MBq) & 0.14 & 0.19 & 0.43 & 0.013 & 0.017 & 0.037 \\
\hline
\end{tabular}

Tabella 2. Dose assorbita dagli organi principali e dose efficace in corso di scintigrafia con ${ }^{131}$ I-MIBG O ${ }^{123}$ I-MIBG (modificata da: Bombardieri E et a. ${ }^{131} \mathrm{I}^{123} \mathrm{I}$-Metaiodobenzylguanidine (mIBG) scintigraphy: procedure guidelines for tumour imaging. Eur J NucIMedMol Imaging2010; 37: 2436-46. Si rimanda a questa fonte per $i$ dati relativi agli altri organi 
150,000 counts for each field of view, $128 \times 128$ matrix. For 123I-MIBG: whole-body images with anterior and posterior acquisitions and velocity of $5 \mathrm{~cm} /$ minute or anterior and posterior planar images of the head, neck, thorax, abdomen, pelvis, arms and lower limbs: at least 500,000 counts for each field view, $256 \times 256$ or 10 'acquisition matrix. In children with neuroblastoma it is advisable to acquire images of the head in both anterior and lateral projection. SPECT can improve diagnostic accuracy and is especially useful when there are interpretative doubts about the location of the lesions and the interpretation of radiopharmaceutical uptake. It should be performed in all cases, including pediatric patients who however may require sedation. Parameters: $360^{\circ}$ of rotation, 120 total projections, $25-35 \mathrm{sec}$ per step, $128 \times 128$ or $64 \times 64$ matrix. Co-registration of CT images can be used to correct attenuation and to better define the location of the lesions. It is particularly useful in this type of exam. Processing Planar images do not require special processing. For SPECT images it is important to take into account the different types of equipment and software available.

\subsection{General neuroblastoma}

Neuroblastoma is responsible for more than $7 \%$ of malignant tumors in people under the age of 15 and about $15 \%$ of all pediatric cancer deaths. This is the most common extracranial tumor in pediatrics and the most frequently diagnosed tumor in childhood.

In the 1980s, when it was possible to demonstrate cancer through the search for catecholamines in the urine, screening programs were undertaken: a Japanese study, performed on urine collected in children at 6 months of age, seemed to show that an early diagnosis it could influence the evolution of the disease. But two prospective clinical trials in the United States and Germany subsequently demonstrated that screening does not reduce lethality (Schilling FH et al., 2002; Woods WG et al, 2002), for which it was abandoned.

It is a disease of the sympathetic-adrenal line of the neural crest, whereby tumors can develop anywhere in the sympathetic neural system. Most primary tumors are located in the abdomen, half of them in the adrenal medulla. Other common sites of the disease include:

- The neck

- Chest

- The pelvis.

Presenting signs and symptoms are highly variable and depend in part on the location of the primary tumor, but also on the presence or absence of metastases or paraneoplastic syndromes. While there may be overlaps, there are three possible scenarios:

\subsection{Localized cancer}

About $40 \%$ of patients present with localized disease, which can range from the casual discovery of an adrenal mass to prenatal ultrasonography to very large and locally invasive tumors along the sympathetic chain in later ages. Primary thoracic tumors can also be found incidentally on a chest X-ray, while cervical masses can be associated with Horner's syndrome (ptosis of the upper eyelid + myosis + narrowing of the palpebral fissure). Paraspinal tumors of the thorax, abdomen and pelvis are found in 5-15\% of patients: these tumors can extend to the intervertebral neural foramina (hourglass neuroblastomas), causing compression symp- toms of the nerve roots and spinal cord. About $5 \%$ of all neuroblastomas are diagnosed in patients who have neurological signs related to involvement of the spinal cord, with fatigue, pain and loss of sensation. Localized tumors generally respond to chemotherapy treatment and / or laminectomy: Since laminectomy can have distant consequences, most researchers recommend chemotherapy as the intervention of choice for symptomatic paraspinal neuroblastomas. Two important paraneoplastic syndromes are observed in these patients: intractable watery diarrhea, which ceases with the removal of the tumor, and opsoclonus-myoclonus syndrome, seen in $2-4 \%$ of patients with neuroblastoma: it consists of rapid eye movements, ataxia and irregular muscle movements. In these cases the prognosis is favorable.

\subsection{Metastatic disease}

About half of the patients present with hematogenous metastases. Metastases can be distant, such as in the cortical part of the bones, in the medulla, in the liver and in non-contiguous regional lymph nodes, or by spread of the primary tumor into adjacent lymph nodes. Unlike what is observed in children with localized tumors, these children with metastatic tumors appear deeply ill at the time of diagnosis. These tumors have an inexplicable tendency to metastasize into the bones of the orbit and cause bruising of the orbits and proptosis. Sometimes these patients have renin-mediated hypertension due to impaired renal vascularity. Spread to the central nervous system can be observed as the disease evolves.
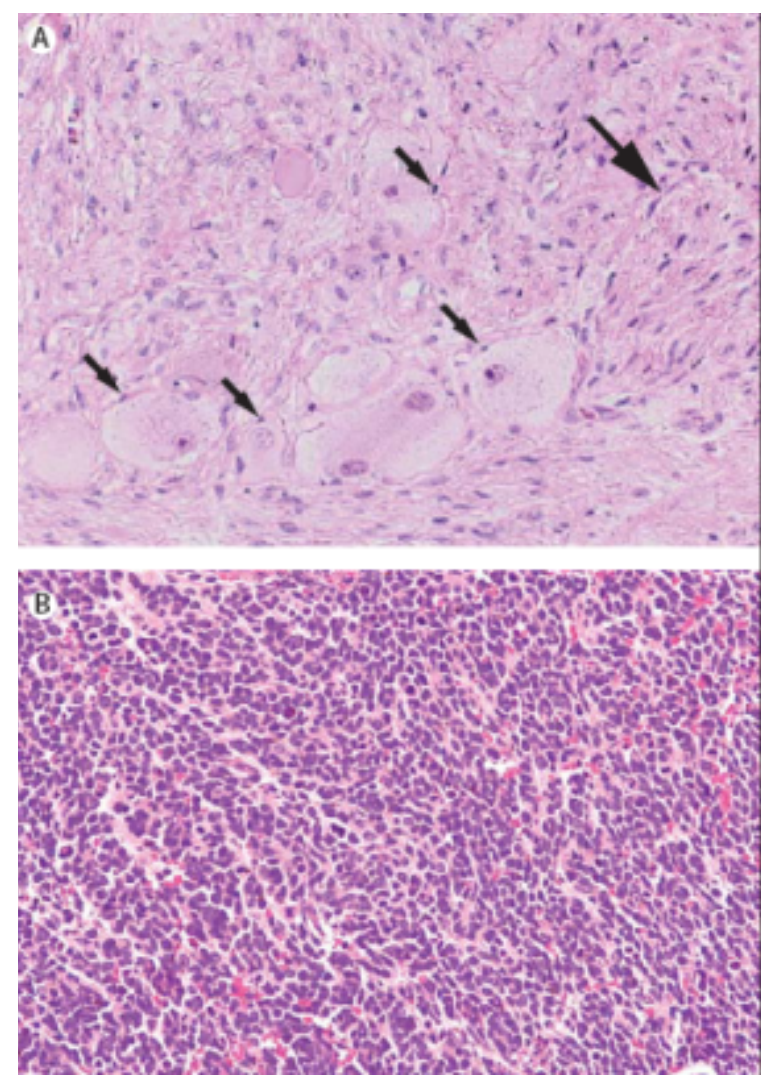

Fig. 19 - Metastatic tumors with varying degrees of differentiation.

a) Schwann cells and ganglion cells (indicated by arrows) are abundant in stroma-rich neuroblastoma

b) in neuroblastomas poor in stroma, rounded, crammed cells with scarce cytoplasm are evident. 


\subsection{4 s disease}

D'Angio and coll. first described the characteristics of this form which occurs in 5\% of patients (D'Angio GJ et al, 1971). These children have a small primary tumor with liver, skin and bone marrow metastases, which almost always regress spontaneously. However, infants less than 2 months of age can have extensive and rapidly progressive liver spread, which can even impair breathing.

\subsection{Diagnosis}

The diagnosis of neuroblastoma is based on the presence of characteristic histopathological features or the presence of tumor cells in the bone marrow aspirate, accompanied by increased concentrations of urinary catecholamines.

Histopathological assessments are essential for determining treatment, especially in children under the age of 18 months (Figure 1). Tumor biopsies are sufficient for genetic molecular analyzes, so these searches should be encouraged at the time of diagnosis.

\subsection{Clinical evaluation of the disease}

Betaiodobenzylguanidine (MIBG) scintigraphy has increased sensitivity and specificity compared to previous tracers. Since MBIG selectively concentrates in more

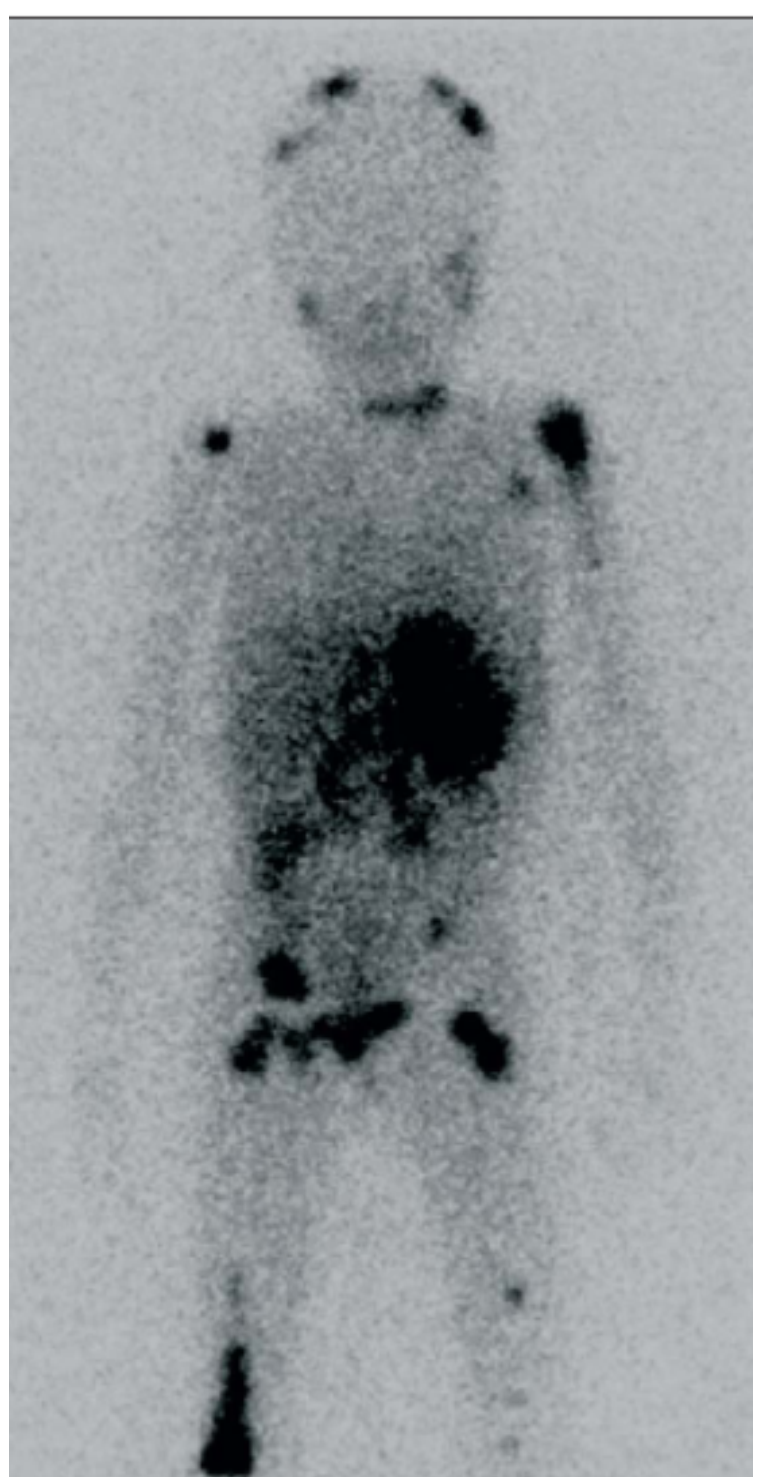

Fig. 20 - Betaiodobenzylguanidine (MIBG) highlights multiple disease sites, including bone and subcutaneous tissue. than $90 \%$ of neuroblastomas, MBIG scintigraphy is a highly specific method for primary tumor assessment and metastatic disease. once joded with 131I or 123I, allows effective imaging of resulting neuroectodermal tumors, including neuroblastomas and pseochromocytomas [2-6] although other tumors, such as paragangliomas, medullary thyroid carcinomas, carcinoid tumors, cell tumors Merkel's skin and metastases from these cancers have been shown to take over MIBG $[7,8]$.

Since a number of highly undifferentiated neuroblastomas may not concentrate MBIG, TC-diphosphonate can be used in bone research when MBIG is negative or unavailable.

MBIG is also recommended for re-evaluation during and after therapy in high-risk patients who tested positive for MBIG at diagnosis.

Bone marrow disease should also be evaluated by other means, such as bone marrow aspirates and biopsies.

Risk assessment depends on many clinical and biological aspects. As risk assessment varies greatly from country to country, in the past two years the International Neuroblastoma Risk Group (INRG) has reviewed the medical records of 11,054 children, treated in Europe, Japan, Canada, USA and Australia between 1974 and 2002. A consensus was thus obtained:

- Regarding age: before and after 18 months;

- Stadium

- $\quad$ The MYCN status

The International Neuroblastoma Staging System has developed the staging criteria, which have been accepted around the world (see Table). These studies have shown that the radiological characteristics of the primary tumor are useful in predicting the best and most successful surgical treatment. Loco-radiological aspects were used to distinguish locoregional tumors that do not involve local structures (INRG stage L1) from locally invasive tumors (INRG stage L2). Stages $\mathrm{M}$ and MS have been proposed to categorize tumors that are extensively metastatic or that have $4 \mathrm{~S}$ behavior. As regards age, it is preferable to take into account the age of 15 to 18 months, instead of that of 12 months. The presence of multiple copies of MYCN can be observed in tumor cells using the MYCN labeled probe.

\subsection{Indications}

The main indications concern:

- Confirmation in suspected neuroectodermally arising tumors, including neuroblastoma,

- Phaochromocytoma and ganglioneuroma.

- $\quad$ Staging of the disease.

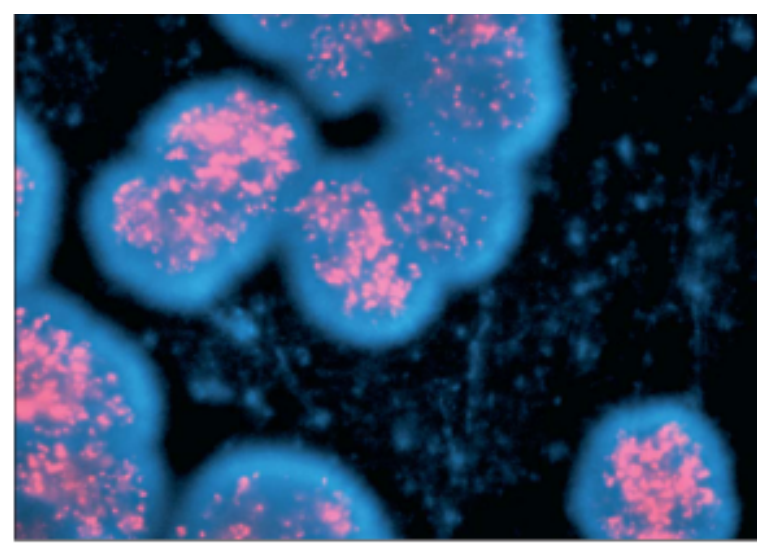

Fig. 21 - MYCN amplification demonstrated by fluorescence in in situ hydridization (FISH) (Maris, JM, 2007) 


\begin{tabular}{|l|l|}
\hline Stadium & Characteristics of the tumor \\
\hline 1 & $\begin{array}{l}\text { Localized tumor with complete gross excision, with or without residual microscopic disease, represented } \\
\text { by microscopically negative ipsolateral lymph nodes for tumor (lymph nodes adhering to the tumor and } \\
\text { removed with the primary tumor may be positive). }\end{array}$ \\
\hline 2A & $\begin{array}{l}\text { Localized tumor with incomplete gross excision, with non-adherent ipsolateral lymph nodes, positive for } \\
\text { tumor. }\end{array}$ \\
\hline 2B & $\begin{array}{l}\text { Localized tumor with or without gross complete excision, with tumor-positive non-adherent ipsolateral } \\
\text { lymph nodes. Enlarged contralateral lymph nodes should be microscopically negative. }\end{array}$ \\
\hline 3 & $\begin{array}{l}\text { Unilateral unresectable tumor with or without involvement of regional lymph nodes or unilateral localized } \\
\text { tumor involving regional versus lateral lymph nodes or midline tumor with bilateral extension of infiltration } \\
\text { (unresectable) or involving lymph nodes }\end{array}$ \\
\hline 4 & $\begin{array}{l}\text { Any primary tumor with spread to distant lymph nodes, bone, bone marrow, or other organs (except stage } \\
4 \mathrm{~S}),\end{array}$ \\
\hline $4 \mathrm{~S}$ & $\begin{array}{l}\text { Primary tumor localized in infants younger than one year (as defined by stages 1, 2A and 2B), with limited } \\
\text { dissemination to the skin, liver or bone marrow }(<10 \% \text { malignant cells) }\end{array}$ \\
\hline
\end{tabular}

Tab. 2

\begin{tabular}{|c|c|c|c|c|c|c|}
\hline Stadium & Age & MYCN & Ploidy & Histology & Other & Risk \\
\hline 1 & & & & & & Bass \\
\hline $2 \mathrm{~A} / 2 \mathrm{~B}$ & & $\begin{array}{l}\text { Not amplified } \\
\text { Not amplified } \\
\text { Not amplified } \\
\text { Amplified }\end{array}$ & & & $\begin{array}{l}>50 \% \text { resection } \\
<50 \% \text { resection } \\
\text { Biopsy only }\end{array}$ & $\begin{array}{l}\text { Bass } \\
\text { Intermediate } \\
\text { Intermediate } \\
\text { High }\end{array}$ \\
\hline 3 & $\begin{array}{l}<547 \text { days } \\
\geq 547 \text { days } \\
\geq 547 \text { days }\end{array}$ & $\begin{array}{l}\text { Not amplified } \\
\text { Not amplified } \\
\text { Amplified } \\
\text { Not amplified }\end{array}$ & & $\begin{array}{l}\text { Favorable } \\
\text { Unfavorable }\end{array}$ & & $\begin{array}{l}\text { Intermediate } \\
\text { Intermediate } \\
\text { High } \\
\text { High }\end{array}$ \\
\hline 4 & $\begin{array}{l}<365 \text { days } \\
<365 \text { days } \\
365-547 \text { days } \\
365-547 \text { days } \\
365-547 \text { days } \\
365-547 \text { days } \\
\geq 547 \text { days }\end{array}$ & $\begin{array}{l}\text { Amplified } \\
\text { Not amplified } \\
\text { Amplified } \\
\text { Not amplified }\end{array}$ & $\begin{array}{l}\mathrm{DI}=1 \\
\mathrm{DI}>1\end{array}$ & $\begin{array}{l}\text { Unfavorable } \\
\text { Favorable }\end{array}$ & & $\begin{array}{l}\text { High } \\
\text { Intermediate } \\
\text { High } \\
\text { High } \\
\text { High } \\
\text { Intermediate } \\
\text { High }\end{array}$ \\
\hline $4 \mathrm{~S}$ & $\begin{array}{l}<365 \text { days } \\
<365 \text { days } \\
<365 \text { days } \\
<365 \text { days } \\
<365 \text { days } \\
<365 \text { days }\end{array}$ & $\begin{array}{l}\text { Not amplified } \\
\text { Not amplified } \\
\text { Lost } \\
\text { Not amplified } \\
\text { Not amplified } \\
\text { Amplified }\end{array}$ & $\begin{array}{l}\mathrm{DI}>1 \\
\mathrm{DI}=1 \\
\text { Lost }\end{array}$ & $\begin{array}{l}\text { Favorable } \\
\text { Lost } \\
\text { Unfavorable }\end{array}$ & $\begin{array}{l}\text { Asymptomatic } \\
\text { Symptomatic }\end{array}$ & $\begin{array}{l}\text { Bass } \\
\text { Intermediate } \\
\text { Intermediate } \\
\text { Intermediate } \\
\text { Intermediate } \\
\text { High }\end{array}$ \\
\hline
\end{tabular}

Tab. 3 - INSS staging

- Control of neuroblastoma under chemotherapy, particularly in stage IV and IV patients.

- Before and after primary tumor surgery.

- Follow-up after treatment to rule out subclinical relapse, especially in the bone marrow and also in

- The case of any clinical abnormalities during follow-up, especially bone pain.

- Before programming MIBG therapy

\subsection{Contraindications}

Absent

\subsection{Procedure}

Referring physicians should be advised that radioiodated MIBG is a cyclotronic product and therefore not Always available at all times. Several days are required to obtain the MIBG even there is a period (days) Between injection and scan depending on which iodine nuclide is used.

\section{Thyroid Pediatric Scintigraphy}

It is a method that allows to obtain the functional map of the distribution of a radiopharmaceutical within the thyroid. It provides the doctor with essential information on: function, size and homogeneity of the gland and any palpable nodules within it.It is performed with the gamma camera, sometimes with the SPET technique

\subsection{Indications}

- Isolating nodes (Cysts, true or degenerative; Hemorrhages;Normo / hypo-functioning multinodular goiter; Benign and malignant thyroid neoplasms; Parathyroid adenomas and carcinomas;Thyroiditis)

- Hyper-catching nodes (Autonomous adenoma ;Hyper-functioning Multinodular goiter; Neoplasms in hyper-functioning node ; Thyroiditis)

- Initial diagnostic classification of almost all thy- 


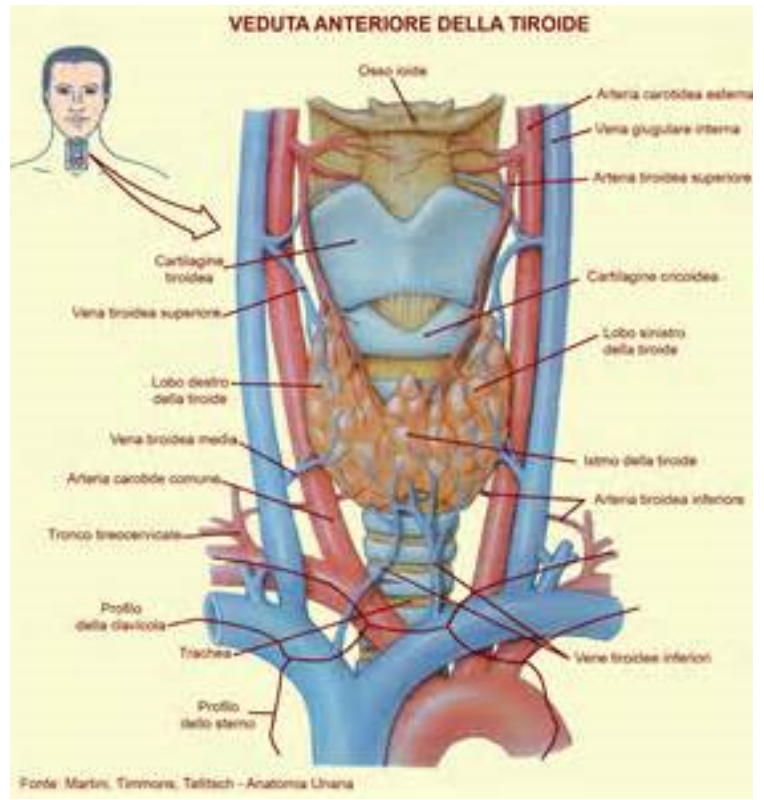

Fig. 22 - Anatomical representation of the thyroid gland

roid diseases

- Control after partial or total thyroidectomy

- Before any radio-iodine therapy

- In the follow-up of thyroid diseases only if necessary

16.2 Main thyroid disorders in pediatric patients 16.2.1 Congenital hypothyroidism

A fairly common pathology affecting the thyroid gland in childhood is congenital hypothyroidism, which represents one of the most common treatable causes of mental retardation. It affects about 1 in 3000-4000 newborns .

Congenital hypothyroidism is pauci or asymptomatic at birth and was clinically recognized in the first months of life, when irreversible damage to psychomotor development had already occurred.

Congenital hypothyroidism (ie present at birth) can be transient or permanent. The symptoms and signs of congenital hypothyroidism usually become manifest in the first months of life.

Prolonged jaundice, prolonged gestation beyond 42 weeks, edema, feeding difficulties, delayed emission of meconium, abdominal distension, hypothermia, respiratory distress may be present at birth. A large anterior fontanel and posterior fontanel $>0.5 \mathrm{~cm}$ in diameter may be present.

Subsequently the child appears hypotonic, sleepy, poorly reactive to lethargy, with feeding difficulties, hoarse and scanty crying, dry, cold and marbled skin. umbilical hernia, large tongue, constipation, poor growth.

The etiology of hypothyroidism with thyroid scintigraphy and / or ecocolordoppler must be specified.

The scintigraphy with the best sensitivity is that with Iodine-123, which is organized and allows to visualize both ectopic thyroid tissue and hormonogenesis defects. It has the disadvantage of being expensive and not available in many centers, so Technetium 99 is often used. Iodine-131 should not be used.

Echocolordoppler is useful both as an investigation that confirms the possible agenesis-ectopy of the thyroid, and with modern devices, as a less invasive and expensive method, performed in place of scintigraphy.

It is important to underline that the execution of the imaging methods must in no case delay the initiation of replacement therapy with L-thyroxine.

\subsubsection{Congenital hypertiroidism}

Another important pathology affecting the thyroid gland is congenital hyperthyroidism. Most cases of hyperthyroidism diagnosed at birth are due to the transplacental passage of TSH receptor-stimulating antibodies. The duration of hyperthyroidism in the newborn is therefore linked to the persistence of circulating antibodies The symptomatology may be present at birth, normally occurs during the first week but can appear within 1-2 months

Hyperthyroidism can be suspected in pregnancy due to fetal tachycardia ( $>160$ beats / minute). In the newborn there is tachycardia, irritability, poor weight gain, ocular protrusion, "wide open" gaze. Goiter may be present which can cause compression of the trachea during delivery.

Untreated fetal and neonatal hyperthyroidism can cause craniosynostosis, poor growth and mental retardation. Symptoms may persist for a few months (usually 2-3).

\subsubsection{Permanent neonatal hypertiroidism}

It is due to a mutation in the TSH receptor gene and is activated even in the absence of TSH, producing a state of hyperthyroidism. It's rare. Most cases are transmitted with an autosomal dominant trait, but can be sporadic, due to the onset of new mutations. The therapy is ablative, surgical or, depending on the age, with radioiodine.

\subsection{Thyroid pathology in children and teenagers}

\section{Hypothyroidism}

Chronic lymphocytic thyroiditis

It was described in 1912 as Hashimoto's thyroiditis.

Chronic lymphocytic thyroiditis (CLT) is the most frequent cause of hypothyroidism in children and adolescents. It is an autoimmune disease of the thyroid gland, characterized by an immune reaction of the organism against its own constituent, which manifests itself with the lymphocytic infiltration of the thyroid gland and in most cases with the appearance of autoantibodies directed against thyroid antigens (antibodies anti-thyroperoxidase, ab-TPO), (anti-thyroglobulin antibodies, ab-Tg) detectable in the blood.

CLT does not cause hypothyroidism in all subjects, and thyroid function can remain normal for many years. In rare cases it can give transitory or persistent forms of hyperthyroidism. The transient forms are due to tissue damage of the thyroid with massive release of thyroid hormones in circulation, the persistent forms (called Hashitoxicosis) are mixed forms with autoimmune hyperthyroidism (Graves' disease) which is caused by antibodies against the TSH receptor (TRAb) which stimulate the production of thyroid hormones and which are also detectable in Hashitoxicosis.

CLT can be associated with other autoimmune diseases, such as type 1 diabetes, celiac disease, Addison's disease.

It is more frequent in women and has a peak of onset in pubertal age, but it can also occur in childhood.

Autoimmune diseases of the thyroid gland, both CLT and Graves' disease, are pathologies with a strong family aggregation, that is, they occur with a higher frequency within a family in which a case is diagnosed, compared to the general population. From the point 
of view of genetic predisposition they are considered multifactorial diseases.

CLT can present with goiter, normal volume or more rarely atrophic thyroid.

Thyroid dysgenesia and hormonogenesis disorders Congenital hypothyroidism in Italy is diagnosed at birth with newborn screening. This does not happen in different parts of the world, not only in developing countries but also for example in Eastern European countries.

It may therefore be necessary to make a diagnosis of thyroid dysgenesis in the older child.

Disorders of hormonogenesis can become symptomatic in the child and / or be diagnosed by the appearance of goiter.

Drugs that can interfere with thyroid function include: some anticonvulsants, lithium, amiodarone, aminoglutethymide, sertraline, aminosalicylic acid, as well as antithyroid drugs used in the treatment of hyperthyroidism (propylthiuracil and methimazole).

\section{Iodine deficiency}

It is a major cause of endemic hypothyroidism for approximately 800 million people living in developing countries, rarely in the Western world.

\section{Results of surgical intervention or radiotherapy}

Partial thyroidectomy can cause hypothyroidism. The irradiation of the neck carried out in the therapy of many childhood cancers can 'over the years result in hypothyroidism.

Hypothyroidism in children manifests itself subtly and can also be diagnosed with years of delay for example a deceleration of the growth rate accompanied by a delay in skeletal and dentition maturation. The classic symptoms of hypothyroidism are dry skin, thin and sparse hair, cold intolerance, constipation, periorbital edema. The thyroid can be increased in volume and consistency. In rare cases of very long-lasting hypothyroidism, the sella turcica can be enlarged due to hypertrophy of the pituitary gland (saddle cherry).

There can be pubertal delay but also an early pseudopubertia due to the action of TRH and TSH on the gonadotropin-gonad axis, menstrual irregularities.

Ultrasonography is the examination of choice to define the morphology, the echostructure, the possible presence of nodules and lymph nodes. For therapy,

the replacement dose is about $100 \mathrm{mcg}$ of L-thyroxine per square meter of body surface area, or 5-6 mcg / kg between 1-5 years, 3-4 mcg / $\mathrm{kg}$ between 6-10 years, 2-3 mcg / kg after 11 years. Many children compensate with a lower dosage and therapy must always be adapted to the individual patient in order to maintain TSH values in the average range of normal. In severely hypothyroid children, correction of hypothyroidism is done slowly.

\section{Graves disease}

Graves' disease is responsible for approximately 95\% of pediatric hyperthyroidism cases. It is an autoimmune disease closely related to the previously described chronic lymphocytic thyroiditis (CLT).

Fortunately, it is a rare disease in pediatric age, in fact it has an incidence of 0.8 cases per 1,000,000 inhabitants between 0 and 15 years. The forms that appear in prepubertal age are generally more aggressive and more difficult to treat than those that occur in adolescence.
Hyperthyroidism in Graves' disease is due to the presence in the circulation of particular antibodies, called anti-TSH receptor antibodies (TRAb), which have the ability to bind to the TSH receptor on thyroid cells. This link stimulates the synthesis of thyroid hormones in a completely independent way from the hypothalamus-pituitary regulation, which, through a complex feedback system, keeps the circulating levels of thyroid hormones constant. In Graves' disease, on the other hand, there is a worsening hyperthyroidism, due to the continuous stimulation of TRAb.

These children present hyperactivity, irritability, emotional lability, disturbances in school behavior, difficulty concentrating, changes in writing, sleep disturbances, enuresis, intolerance to heat, weight loss despite increased appetite. the number of bowel movements, tremors, tachycardia. Increased blood pressure. The appearance of the goiter, which may be small, is often not noticed for a long time. Signs of ophthalmopathy may be present (wide gaze, retraction of the upper eyelid, etc., with or without true exophthalmos).

Diagnosis is made by means of scans. Scintigraphy is rarely necessary for differential diagnosis (eg hyperfunctioning nodules).

Graves' disease therapy can be medical, surgical, or radioiodine.

In pediatric age, drug therapy is also used for years, which is not, however, free from possible side effects, even serious ones.

\section{Goiter}

Goiter refers to an increase in the volume of the thyroid, which may be due to stimulation of the thyroid tissue, infiltration or inflammation. The increase in volume can 'be widespread or affect only a part of the thyroid. The most frequent cause of goiter is chronic lymphocytic thyroiditis, (not considering the goiter present in areas with endemic iodine deficiency.

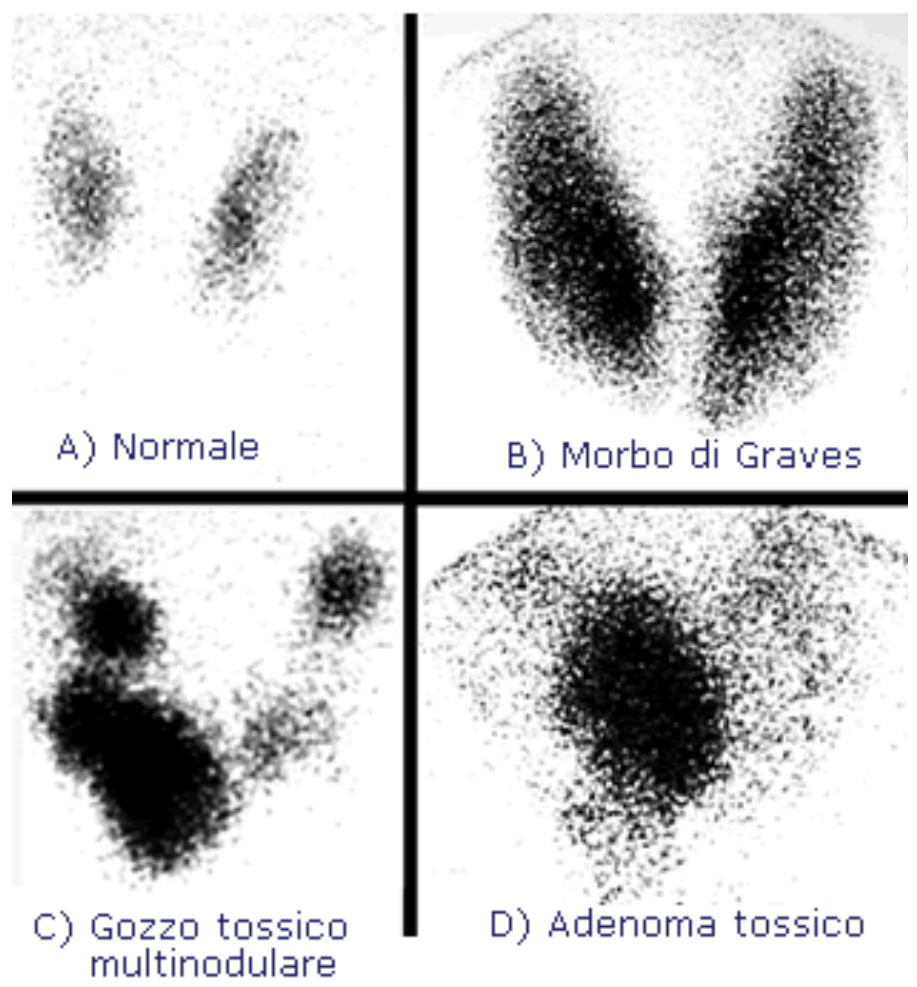

Fig. 23 - Scintigraphy various cases 


\section{SCINTIGRAFIA E NODULAZIONI TIROIDEE}

Nodulo freddo destro

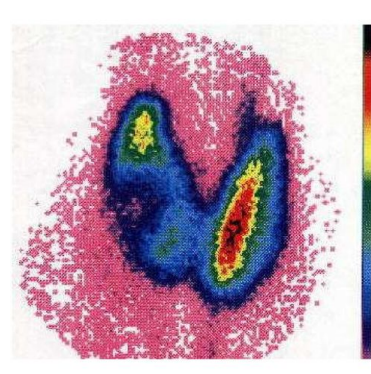

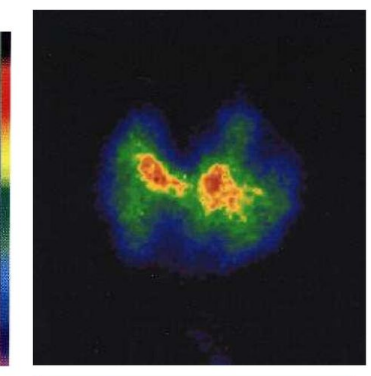

Noduli a vario grado
Fig. 24 - visualization of nodules in scintigraphic image

It is defined idiopathic when its origin is not known. It is thought to be multifactorial in origin, with a genetic component. The increase in thyroid volume would be necessary to ensure sufficient production of thyroid hormones.

\section{Gozzo from other causes}

The thyroid may be enlarged because it is the site of a tumor of a different origin from the thyroid tissue, for example a lymphoma. Or site of localization of other diseases, such as histiocytosis.

Rapid goiter growth must always be a warning sign.

Malformations, cysts, benign and malignant tumors are other causes of thyroid enlargement.

\section{Infectious thyroiditis}

The thyroid can be affected by both bacterial and viral infections. These are rare pathologies in pediatric age. They can be preceded by an upper respiratory infection, such as tonsillitis, pharyngitis and be caused by various pathogens. Acute bacterial suppurative thyroiditis is manifested by a painful, sharp swelling of the anterior region of the neck, fever, chills. The skin may be red and there may be lymphadenopathy. You have a sore throat with difficulty in swallowing. The pain can be violent and accentuated with hyperextension of the neck. The presence of malformations such as the persistence of the piriform sinus favors the infection of the left lobe, and is - after the resolution of the acute episode - surgically corrected to avoid recur-

Sample Pediatric Radiopharmaceutical Calculations

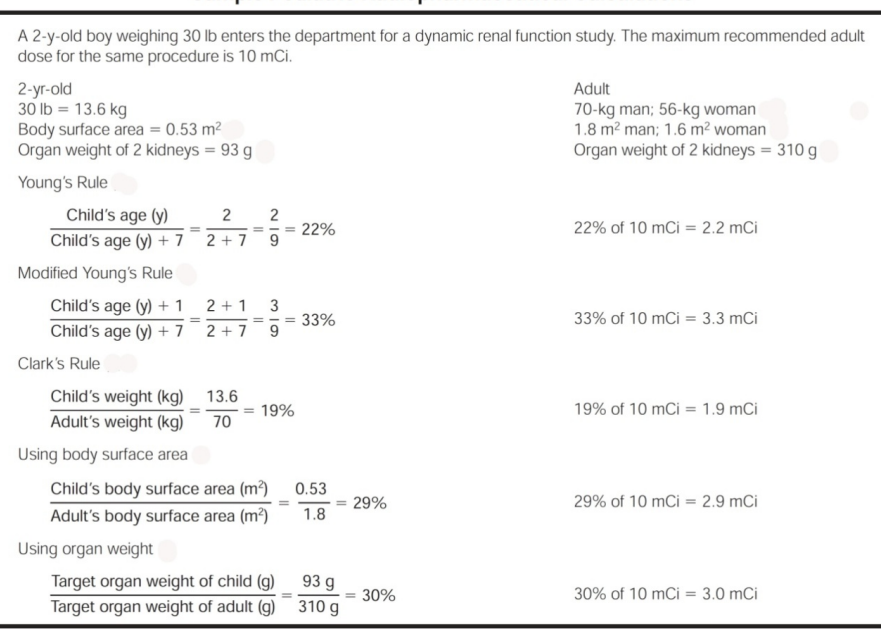

Tab. 4 - Pediatric radiopharmaceutical calculations rence. The therapy is antibiotic. Any appearance of abscesses should be monitored with ultrasound. Subacute thyroiditis is often of viral origin. Therapy is usually symptomatic, with analgesics and non-steroidal antiinflammatory drugs.

\section{Nodules and tumors of the thyroid}

The incidence of thyroid nodules in pediatric age is reported to be $1-1.5 \%$, but is probably underestimated. Predisposing factors for the onset of nodules are female sex, age of puberty, familiarity with thyroid disease, thyroid disease.

The risk of a lump in a child being malignant in nature is 4 times greater than in adults. Consequently, every thyroid nodule in the child must be evaluated to rule out the presence of a neoplasm. Thyroid carcinomas are the most common endocrine tumors in pediatric age and represent $0.5-3 \%$ of all malignancies.

Most pediatric malignant thyroid tumors are differentiated papillary carcinomas. Follicular carcinomas represent about $18 \%$. The thyroid is also frequent site of second tumors induced by other therapies, such as radiation therapy, (eg for Hodgkin's lymphoma, brain tumors, bone marrow transplant). Thyroid tumors can appear in the context of autoimmune thyroiditis.

\section{Medullary carcinoma of the thyroid}

It represents $5 \%$ of thyroid cancers in pediatric age. It is part of two genetic syndromes, MEN (Multiple endocrine neoplasia) and FTMC (familial medullary thyroid carcinoma). Two types of MEN are known. The first MEN 2A is characterized by pheochromocytoma, hyperparathyroidism and medullary thyroid carcinoma. The second MEN 2B, with marfanoid appearance, multiple mucous neuromas of the alimentary and subconjunctival tract, medullary thyroid carcinoma earlier and more aggressive than MEN 2A.

The prognosis of differentiated thyroid tumors is in a good pediatric age, even in the presence of metastases at diagnosis. At 10 years mortality is calculated around $1 \%$

The suspicion or diagnosis of MEN requires evaluation of the genetic mutations implicated in these diseases (RET gene) and prophylactic thyroidectomy in subjects carrying the mutation within 5 years of life for MEN 2A, spent within the first year of life for the MEN $2 \mathrm{~B}$.

\subsection{Radio drug and dosimetry}

Sodium pertechnetate is the radiopharmaceutical of choice for pediatric thyroid imaging, but technetium pertechnetate (Tc $99 \mathrm{mO}-4)$ is preferred.

The dose administered to pediatric patients is $10 \mathrm{MBq}$ intravenous.

\subsection{Examination technique}

After the intravenous administration of the radiopharmaceutical, 10-15 minutes are waited, after which the acquisition begins.

The equipment chosen is the gamma camera with pinhole collimator. An anterior projection is performed with the collimator at a distance of $5-10 \mathrm{~cm}$ from the cutaneous plane in correspondence with the thyroid, so as to have the maximum magnification and the maximum power of resolution of the image.

The acquisition time of the static image is about 5 minutes and the image obtained shows a higher count in the hyperactive areas and lower in the less functional ones. 


\begin{tabular}{|l|l|l|}
\hline EXAM & RADIO DRUG & LDR (MBq) \\
\hline Thyroid uptake & ${ }^{123}$ I-iodide & 2 \\
\hline “ & ${ }^{131}$ I-iodide & 0.37 \\
\hline Thyroid scintigraphy & ${ }^{123}$ I-iodide & 20 \\
\hline “ & ${ }^{99 m}$ Tc-pertechnetate & 110 \\
\hline Renal scintigraphy & ${ }^{99 m}$ Tc-DMSA & 160 \\
\hline “ & ${ }^{99 m}$ Tc-DTPA & 200 \\
\hline “ & ${ }^{99 m}$ Tc-MAG3 & 160 \\
\hline “ & ${ }^{123}$ I-hippuran & 74 \\
\hline $\begin{array}{l}\text { Evaluation of the ectopic gastric } \\
\text { mucosa }\end{array}$ & ${ }^{99 m}$ Tc-pertechnetate & 185 \\
\hline Lung perfusion scan & ${ }^{99 m}$ Tc-MAA & 160 \\
\hline Ventilatory lung scan & ${ }^{99 m}$ Tc-aerosol & 1100 in the nebulizer; 5 'max \\
\hline “ & ${ }^{133}$ Xe & nebulization \\
\hline Bone or joint scan & ${ }^{99 m}$ Tc-diphosphonates & 550 \\
\hline “ & “ & $600<30$ years \\
\hline " & ${ }^{*}$ & 90030 -50 years \\
\hline Tome bone scan or polyphasic scan & 90 years \\
\hline
\end{tabular}

Tab. 5 - In vivo nuclear medicine diagnostics: LDR

\begin{tabular}{|l|l|l|l|l|l|}
\hline $\begin{array}{l}\text { BODY } \\
\text { MASS } \\
\text { Kg }\end{array}$ & $\begin{array}{l}\text { \% OF THE } \\
\text { ACTIVITY } \\
\text { ADMINISTERED } \\
\text { TO THE ADULT }\end{array}$ & $\begin{array}{l}\text { BODY } \\
\text { MASS Kg }\end{array}$ & $\begin{array}{l}\text { \% OF THE ACTIVITY } \\
\text { ADMINISTERED TO } \\
\text { THE ADULT }\end{array}$ & $\begin{array}{l}\text { \% OF THE } \\
\text { MASS Kg } \\
\text { ACTIVITY } \\
\text { ADMINISTERED } \\
\text { TO THE ADULT }\end{array}$ \\
\hline 3 & 10 & 22 & 50 & 42 & 78 \\
\hline 4 & 14 & 24 & 53 & 44 & 80 \\
\hline 6 & 19 & 26 & 56 & 46 & 82 \\
\hline 8 & 23 & 28 & 58 & 48 & 85 \\
\hline 10 & 27 & 30 & 62 & 50 & 88 \\
\hline 12 & 32 & 32 & 65 & $52-54$ & 90 \\
\hline 14 & 36 & 34 & 68 & $56-58$ & 95 \\
\hline 16 & 40 & 36 & 71 & $60-70$ & 100 \\
\hline 18 & 44 & 38 & 73 & & \\
\hline 20 & 48 & 40 & 76 & & \\
\hline
\end{tabular}

\section{Talb. 6}

\section{PEDIATRIC}

RADIOPHARMACEUTICAL

CALCULATIONS IN PEDIATRIC

NUCLEAR MIEICINE AND LDR

Compared to nuclear medicine for adults, different procedures and strategies are needed for pediatric nuclear medicine, this is due to physiological differences between children and adults.

These differences also affect the dosimetry and the activity of the radiopharmaceutical to be administered to the pediatric patient. In particular, each department must choose a precise calculation criterion to be used and calculate the dose consistently, also based on the machinery available to them. Routine calculations use the patient's age, weight, or body surface area to calculate the amount of activity that should be administered to a pediatric patient. Table 4 provides a sample of cal- culations based on different formulas.

Sometimes the calculated activity is not sufficient to obtain quality images and sometimes the calculated activity does not correspond with the dosimetric limits of the radiation.

It is therefore advisable to always have as a reference the so-called LDR (diagnostic reference levels), or "dose levels in medical radiodiagnostic practices or, in the case of diagnostic nuclear medicine, activity levels, for typical tests for groups of patients of standard build or standard phantoms for widely defined types of equipment. These levels should not be exceeded for standard procedures, in conditions of correct and normal applications regarding the diagnostic and technical intervention ". The LDRs refer to an adult, with a body mass of no less than $60 \mathrm{~kg}$, presumably free from significant alterations in the metabolism and / or 
elimination of radiopharmaceuticals. Table 5 provides the reference data according to the LDR for some scintigraphic examinations:

In case of body mass less than $60 \mathrm{~kg}$ and in particular in children it is recommended to reduce the activity administered according to the scheme shown in table 6 (EANM - European Association of Nuclear Medicine):

\section{CONCLUSIONS}

Nuclear medicine is a medical specialty which, like radiology, is based on the diagnostic use of low amounts of radiation. The term "nuclear" derives from the use of radiation emitted by the nuclei of some artificially produced isotopes for medical purposes, the most widely used of which today is Technetium $99 \mathrm{~m}$. Radionuclides are bound to numerous chemical substances (consequently called radiolabeled) which, after their administration, become part of the metabolic processes in a similar way to those assumed physiologically. By means of the appropriate instrumentation it is therefore possible to localize the distribution of the radiocompound, following its path within the organism, "photographing" the organ or apparatus under examination and at the same time evaluating its functional attitude. In this way the images come to represent the morphological expression of a function Nuclear medicine therefore makes it possible to recognize functional alterations, providing information that is often essential for defining diagnosis and prognosis, therapies and follow-up. An aspect of not secondary importance is represented by the ability of scintigraphic imaging to highlight an impairment of function even before anatomical alterations are recognizable. In this work I wanted to highlight the relevant aspect represented by the safety of these investigations: the amount of radiation is in fact so modest that diagnostic procedures can also be performed in pediatric age;with the description of the various scintigraphies that can be performed on children.

In the part concerning the general characteristics of nuclear medicine, the components of a gamma camera are reported, the equipment used in nuclear medicine, or the instrument that captures the radiation emitted by the patient.
Pediatric nuclear medicine deals with scintigraphic examinations performed in infants, children and adolescents and it is possible to perform almost all the investigations envisaged for the adult. The differences concern the indications for the execution of the examination, which are specific to pathologies of the pediatric age, such as the diagnosis of congenital childhood disorders or those that develop in the first years of life. However, there are some tests that have the same indications in pediatrics as in adults, for example the evaluation of positivity and extension of tumor lesions of the skeleton through bone scintigraphy.

In the second part of the work, the individual pediatric scintigraphies and some particularly important pathologies were discussed. The scans are therefore analyzed in the various technical aspects: bone with total body and polyphasic technique, perfusion and ventilatory pulmonary, sequential and static renal, thyroid and for the search of ectopic gastric mucosa.

What certainly differentiates pediatric scans from those performed in adults are mainly the doses. In fact, in children the dose administered is lower, as the child can be considered as a "miniature adult".

Of course, pediatric dosimetry is regulated by Legislative Decree no. 187 of 26 May 2000 and, in particular where reference is made to the LDR, or the "diagnostic reference levels", in which, depending on the body weight, the percentage of activity that must be administered to the child is indicated based on that introduced in the adult.

In addition, other methods are mentioned that can be used to calculate the activity to be administered in children, referring to age or weight or body surface or weight of the target organ, often compared with the corresponding ones in adults; but, in any case, they correspond in principle to those published in the LDR.

Often the information provided by scintigraphic examinations is unique and not obtainable with other diagnostic procedures and also much less traumatic. This method is more effective than another procedure and the activity administered is so low that it does not affect vital organs.

\section{REFERENCES}

1. Piepsz A, Colarinha P, Gordon I, Hahn K, Olivier P, Sixt R, van Velzen J; Paediatric Committee of the European Association of Nuclear Medicine. Guidelines for glomerular filtration rate determination in children. Eur J Nucl Med. 2001 Mar;28(3):BP31-6. PMID: 11315614.

2. Piepsz A, Colarinha P, Gordon I, Hahn K, Olivier P, Roca I, Sixt R, van Velzen J; Paediatric Committee of the European Association of Nuclear Medicine. Guidelines for 99mTc-DMSA scintigraphy in children. Eur J Nucl Med. 2001 Mar;28(3):BP37-41. PMID: 11315615.

3. Ciofetta G, Piepsz A, Roca I, Fisher S, Hahn K, Sixt R, Biassoni L, De Palma D, Zucchetta P; Paediatric Committee of the European Association of Nuclear Medicine. Guidelines for lung scintigraphy in children. Eur J Nucl Med Mol Imaging. 2007 Sep;34(9):1518-26. doi: 10.1007/s00259-007-0485-3. PMID: 17602223 
4. Gordon I, Piepsz A, Sixt R; Auspices of Paediatric Committee of European Association of Nuclear Medicine. Guidelines for standard and diuretic renogram in children. Eur J Nucl Med Mol Imaging. 2011 Jun;38(6):1175-88. doi: 10.1007/s00259-011-1811-3. PMID: 21503762. Korean J Pain.2017 Jul; 30 (3): 165-175. doi: 10.3344 / kjp.2017.30.3.165. Epub 2017 Jun 30.

5. Shin SH, Kim SJ. Bone scintigraphy in patients with pain. Korean J Pain. 2017 Jul;30(3):165-175. doi: 10.3344/kjp.2017.30.3.165. Epub 2017 Jun 30. PMID: 28757916; PMCID: PMC5532523.

6. Decreto Legislativo del 26 maggio 2000, n. 187, Article 2, paragraph 1p.

7. Volterrani D, Erba PA, Mariani G. Fondamenti di Medicina Nucleare - Tecniche e Applicazioni. Milan: Springer; 2010. 